\title{
Def-6, a Novel Regulator of Small GTPases in Podocytes, Acts Downstream of Atypical Protein Kinase C (aPKC) $\lambda / \mathrm{t}$
}

Kirstin Worthmann, ${ }^{*}$ Michael Leitges, ${ }^{\dagger}$ Beina Teng, ${ }^{*}$ Marcello Sestu, ${ }^{\ddagger}$ Irini Tossidou, ${ }^{*}$ Thomas Samson, ${ }^{\S}$ Hermann Haller, ${ }^{*}$ Tobias B. Huber, ${ }^{\mathbb{I} \|}$ and Mario Schiffer*

\begin{abstract}
From the Division of Nephrology, * Department of Medicine, Hannover Medical School, Hannover, Germany; the Biotechnology Centre of Oslo ${ }^{\dagger}$ University of Oslo, Oslo, Norway; Faculty of Medicine, ${ }^{\ddagger}$ Interdisciplinary Centre for Clinical Research (IZKF) Leipzig, University of Leipzig, Leipzig, Germany; Department of Cell and Developmental Biology, ${ }^{\S}$ Lineberger Comprehensive Cancer Center, University of North Carolina, Chapel Hill, North Carolina; the Renal Division, $"$ University Hospital Freiburg, Freiburg, Germany; and the BIOSS Centre for Biological Signalling Studies, "Albert-Ludwigs-University, Freiburg, Germany
\end{abstract}

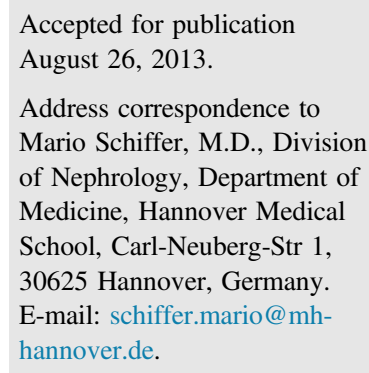

\begin{abstract}
The atypical protein kinase $\mathrm{C}(\mathrm{aPKC})$ isotypes PKC $\lambda / \mathrm{\imath}$ and $\mathrm{PKC} \zeta$ are both expressed in podocytes; however, little is known about differences in their function. Previous studies in mice have demonstrated that podocyte-specific loss of PKC $\lambda / \mathrm{l}$ leads to a severe glomerular phenotype, whereas mice deficient in PKC $\zeta$ develop no renal phenotype. We analyzed various effects caused by PKC $\lambda / \imath$ and PKC $\zeta$ deficiency in cultured

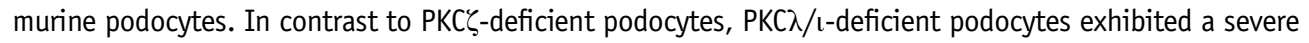
actin cytoskeletal phenotype, reduced cell size, decreased number of focal adhesions, and increased activation of small GTPases. Comparative microarray analysis revealed that the guanine nucleotide exchange factor Def- 6 was specifically up-regulated in PKC $\lambda /$-deficient podocytes. In vivo Def- 6 expression is significantly increased in podocytes of $\mathrm{PKC} \lambda / \mathrm{l}$-deficient mice. Cultured $\mathrm{PKC} \lambda / \mathrm{l}$-deficient podocytes exhibited an enhanced membrane association of Def- 6 , indicating enhanced activation. Overexpression of aPKC $\lambda / \mathrm{i}$ in PKC $\lambda /$ - -deficient podocytes could reduce the membrane-associated expression of Def- 6 and rescue the actin phenotype. In the present study, PKC $\lambda / \mathrm{i}$ was identified as an important factor for actin cytoskeletal regulation in podocytes and Def- 6 as a specific downstream target of PKC $\lambda / \mathrm{I}$ that regulates the activity of small GTPases and subsequently the actin cytoskeleton of podocytes. (Am J Pathol 2013, 183: 1945-1959; http:// dx.doi.org/10.1016/j.ajpath.2013.08.026)
\end{abstract}

The protein kinase C (PKC) family consists of 10 serine/ threonine kinases, which can be divided into three subfamilies on the basis of their different regulatory domains. Conventional PKCs (PKC $\alpha, \mathrm{PKC} \beta \mathrm{I}, \mathrm{PKC} \beta \mathrm{II}$, and $\mathrm{PKC} \gamma)$ and novel PKCs (PKC $\delta, \operatorname{PKC} \theta, \mathrm{PKC} \eta$, and $\mathrm{PKC} \varepsilon$ ) can be activated by calcium or diacylglycerol (DAG) via $\mathrm{C} 1$ and C2 domains. The third subfamily, atypical PKCs (aPKCs) $[\mathrm{PKC} \lambda / \mathrm{\iota}$ (PKC $\lambda$ in mouse and $\mathrm{PKC \iota}$ in human) and PKC $\zeta$ ], lack the calcium-sensitive $\mathrm{C} 2$ domain and possess an atypical $\mathrm{C} 1$ domain that does not bind DAG but binds phosphatidylinositol 3,4,5-triphosphate (PIP3) or ceramide instead. ${ }^{1}$ The amino acid sequences of PKC $\lambda / \mathrm{l}$ and PKC $\zeta$ are $72 \%$ identical $^{2}$; however, the functions of these two proteins in vivo seem to be distinct. Because complete knockout of $\mathrm{PKC} \lambda / \mathrm{s}$ in mice is lethal before embryonic day $9,^{3}$ we created a podocyte-specific $\mathrm{PKC} \lambda / \mathrm{l}$ knockout $\left(P K C \mathrm{~N}^{-1-}\right)$ mouse by mating mice with a floxed $P K C \mathrm{~N}_{\mathrm{l}}$ gene and Podocin-Cre mice ${ }^{4}$ with expression of the Cre recombinase dependent on the podocyte-specific podocin promoter. Podocyte-specific deletion of $\mathrm{PKC} \lambda / \mathrm{s}$ led to a severe glomerular phenotype with glomerulosclerosis, proteinuria, and death at age 4 to 5 weeks. ${ }^{5}$ In contrast to $\mathrm{PKC} \lambda / \mathrm{l}$, complete knockout of $\mathrm{PKC} \zeta\left(P K C \zeta^{-/-}\right)$is rather mild. $P K C \zeta^{-/-}$mice develop no obvious renal phenotype but exhibit changes in their secondary lymphatic organs as a result of a disturbed NF- $\kappa$ B pathway. ${ }^{6}$ Recently, we showed that double-knockout of aPKCs in podocytes leads to a

\footnotetext{
Supported by a grant from the Else Kröner-Fresenius-Stiftung Foundation and by grant EXC 294 from the Excellence Initiative of the German Federal and State Governments (T.B.H.); grants Schi 587/3, 587/4, and 587/6 and grant SFB566 (M.S.); and grant GM029860 to Keith Burridge (principal investigator of T.S.) from the NIH.

T.B.H. and M.S. contributed equally to this work as senior authors.
} 
developmental phenotype with no formation of a normal foot processes network, defective glomerular maturation, incomplete capillary formation, and mesangiolysis. ${ }^{7}$ The severe podocyte foot process effacement in the podocytespecific $P K C \mathrm{~N}^{-1-}$ mice and the inability to form foot processes in the double-knockout mice suggests an important role for the actin cytoskeleton of podocytes. The actin cytoskeleton of podocytes is a highly dynamic and complex structure $^{8-11}$ that also has an important role in maintenance of the slit diaphragm in various renal diseases. ${ }^{12}$ When the actin cytoskeleton of podocyte foot processes changes from parallel bundles of actin ${ }^{13}$ to a dense network of short filaments, foot process effacement and, in turn, proteinuria can develop. ${ }^{14}$ A group of key regulators for actin cytoskeletal regulation are the small GTPases, which are molecular switches that change between a GDP-bound inactive form and a GTP-bound active form. Guanine nucleotide exchange factors (GEFs) control the GDP/GTP exchange by increasing the exchange rate, which leads to activation of small GTPases. ${ }^{15}$ Recent studies have demonstrated the critical involvement of small GTPases in regulation of podocyte actin cytoskeleton in normal podocyte homeostasis and in acquired and genetic glomerular diseases. ${ }^{16-19}$ Herein we demonstrate that differentially expressed FDCP-6 (Def-6), a GEF for the Rho GTPases Rac1 and Cdc $42,{ }^{20}$ is differentially affected by the aPKC isoforms $\lambda / \mathrm{t}$ and $\zeta$ in podocytes. Def- 6 , also known as swap70-like adaptor of T cells or IRF-4-binding protein, consists of an EF-hand motif at the $\mathrm{N}$ terminus, a pleckstrin homology domain, and a Dbl homology-like domain (DHL) at its $\mathrm{C}$ terminus. Def- 6 contains 631 amino acids, has a predicted molecular weight of $74 \mathrm{kD},{ }^{21}$ and exhibits much homology to the B cell-specific GEF swap70. To date, most investigations of the function or expression of Def-6 have been performed in B or T cells because Def-6 is highly expressed in lymphatic organs. ${ }^{21}$ Herein we report for the first time an important role for Def-6 in a renal epithelial cell type.

\section{Materials and Methods}

\section{Plasmids, Antibodies, and Reagents}

The plasmids Def-6 FL (bp 1-2015 in pEGFP-C2) and Def6 DHL (bp 1066-1934 in pEGFP-C3) have been used in previous studies. ${ }^{22}$ Primary antibodies used were mouse monoclonal anti-vinculin (clone hVIN-1) (Sigma-Aldrich Corp., St. Louis, MO), mouse anti-CD29 (integrin $\beta 1$ ) (BD Transduction Laboratories, BD Biosciences, San Jose, CA), goat anti-podocalyxin (R\&D Systems GmbH, Wiesbaden, Germany), mouse monoclonal anti-RhoA, mouse monoclonal anti-Rac1, mouse monoclonal anti-Cdc42 (Cytoskeleton, Inc., Denver, CO), mouse monoclonal anti-Def-6 (Abnova Corp., Taipei, Taiwan), affinity purified rabbit anti-Def-6 (according to a previous study ${ }^{23}$ ), rabbit antiserum anti-Gi $\alpha 3$ (Upstate Biotechnology, Lake Placid, NY), rabbit polyclonal anti-glyceraldehyde-3-phosphate dehydrogenase (GAPDH) (Santa Cruz Biotechnology, Inc., Santa Cruz, CA), rabbit polyclonal anti-Sp1 (Santa Cruz Biotechnology), rat monoclonal anti-entactin/nidogen (Millipore Corp., Billerica, MA), and rabbit monoclonal anti-PKC $\lambda / \iota$ (Cell Signaling Technology, Inc., Beverly, MA). Polyclonal antibody specific for PKC $\zeta$ was raised in rabbits and has been described previously. ${ }^{24}$ All secondary antibodies used for immunostaining were obtained from Invitrogen Corp. (Carlsbad, CA) and included rabbit-Alexa Fluor 488, mouse-Alexa Fluor 488, goat-Alexa Fluor 488, rabbit-Alexa Fluor 555, mouseAlexa Fluor 555, and rat-Alexa Fluor 488. All secondary antibodies used for Western blotting were obtained from Santa Cruz Biotechnology and included goat anti-mouse $\mathrm{IgG}$-horseradish peroxidase and goat anti-rabbit IgG horseradish peroxidase. Further reagents included Alexa Fluor 546 phalloidin (Invitrogen, Darmstadt, Germany), PKCı siRNA, control siRNA (Santa Cruz Biotechnology), aPKC pseudosubstrate and scrambled peptide (BioSource International, Inc., Camarillo, CA), and Def6 (human) recombinant protein (Abnova).

\section{Podocyte-Specific aPKC $\lambda /$ / Knockout Mice}

Generation of podocyte-specific aPKC $\lambda / \mathrm{l}$ knockout mice using floxed $P K C \lambda / \mathrm{t}$ and Podocin-Cre mice has been described previously. ${ }^{5}$ Podocyte-specific aPKC $\lambda / \mathrm{t}$ heterozygous mice were crossed with Immorto transgenic mice. ${ }^{25}$ Homozygous podocyte-specific $\mathrm{aPKC} \lambda / \mathrm{t}$ mice harboring the Immorto transgene were used for generation of immortalized podocyte cell lines.

\section{Isolation of Glomeruli and Establishment of Immortalized Mouse Podocyte Cell Lines}

Glomeruli were isolated from kidneys of 4-week-old mice using a sequential sieving technique as described previously. ${ }^{26}$ Only stable cell lines derived from these glomeruli that were positive for the podocyte-specific markers WT-1 and synaptopodin were used in the experiments.

\section{Podocyte Culture}

Immortalized murine podocytes were cultured as described by Mundel et al. ${ }^{27}$ In brief, cells were cultured on collagen I-coated flasks using RPMI 1640 medium containing 10\% fetal calf serum, $1 \%$ penicillin/streptomycin, and $10 \mathrm{U} / \mathrm{mL}$ $\gamma$-interferon at $33^{\circ} \mathrm{C}$ for proliferation. For differentiation, cells were cultured at $37^{\circ} \mathrm{C}$ without $\gamma$-interferon for 10 to 14 days. For stimulation experiments, cells were serum starved with $1 \%$ fetal calf serum overnight and then treated with 10 $\mu \mathrm{mol} / \mathrm{L}$ aPKC pseudosubstrate for 2 hours at $37^{\circ} \mathrm{C}$ or 10 $\mu \mathrm{mol} / \mathrm{L}$ scrambled peptide, respectively.

\section{Live Cell Microscopy and Cell Movement}

Differentiated deficient and control cells were observed under a microscope (Axio Observer.Z1; Zeiss Microscopy 
GmbH, Göttingen, Germany) at $\times 20$ magnification for 6 hours. Images were obtained automatically every 2 minutes. To quantify the movement of cells, all images were imported into ImageJ software version $1.44 \mathrm{p}$. Using the Manual Tracking plug-in, the nucleus of every selected cell (10 cells of each genotype) was marked on the image sequence. The program measures the distance between two frames, and by summing all distances, the distance covered from the start of recording to the end, at 6 hours.

\section{Transfection}

The day before transfection, podocytes were seeded on glass coverslips and cultured at $33^{\circ} \mathrm{C}$ overnight. Cells were transfected using FuGENE HD transfection reagent (Roche Diagnostics GmbH, Mannheim, Germany) according to the protocol recommended by the manufacturer. Transfection with PKC $\lambda / \mathrm{l}$ siRNA or control siRNA (Santa Cruz Biotechnology) was performed on 3-day differentiated podocytes using lipofectamine 2000 reagent (Invitrogen) according to the manufacturer's protocol. After 6 hours in the incubator, the medium was changed to normal growth medium. After transfection, cells were cultured for 72 hours at $37^{\circ} \mathrm{C}$ and fixed with $4 \%$ paraformaldehyde.

\section{Adenoviral Production and Infection}

Plasmids for adenovirus production were generated using Gateway technology (Invitrogen). Donor vectors were generated by amplifying Green fluorescent protein (GFP)tagged full-length human PKCı or GFP alone with specific primers and cloning into the pDONR221 vector by using BP clonase II enzyme for recombination. Adenoviral constructs were generated using LR Clonase II enzyme, which recombines pDONR221 with pAd/CMV/V5-DEST. All reactions were performed as recommended by the manufacturer (Invitrogen). Adenoviral expression and amplification were performed in HEK293 cells. Podocytes were infected with equal amounts of PKCı-GFP or GFP adenoviral supernatants. After 7 days, podocytes were either harvested for RNA isolation or fixed with $4 \%$ paraformaldehyde for immunostaining.

\section{Polymerase Chain Reaction}

Genomic DNA from cultured podocytes was prepared using the basicdna-OLS Kit (OMNI Life Science GmbH \& Co. KG, Hamburg, Germany) following the protocol recommended by the manufacturer. As controls, genomic DNA samples from tail biopsies were used. PCR was performed under standard conditions in a Primus Thermocycler (MWG-Biotech AG, Ebersberg, Germany) with the following primer pairs: Cre forward, $5^{\prime}$-AGGTTCGTTCACTCATGGA-3'; Cre reverse, 5'-TGCACCAGTTTAGTTACCC-3'; loxP forward, 5'-TTGTGAAAGCGACTGGATTG-3'; loxP reverse, 5'-CTTGGGTGGAGAGGCTATTC-3';
WT reverse, 5'-AATGTTCATGTTCAACACTGCT-3'; Highzeta5 forward, 5'-GCCATCTCCAACAGCCACAG3'; Highzeta5ex, 5'-CCGTTGGCTCGGTACAGCTT-3'; and MO13, 5'-CTTGGGTGGAGAGGCTATTC-3' .

\section{Immunofluorescence Staining}

Immortalized cultured mouse podocytes were seeded on glass coverslips and differentiated for 10 to 14 days. After fixation with $4 \%$ paraformaldehyde, cells were permeabilized with $0.1 \%$ Triton X-100, blocked in $10 \%$ normal donkey serum, and incubated overnight at $4{ }^{\circ} \mathrm{C}$ with the primary antibodies as indicated. After rinsing with PBS, the cells were incubated with a fluorophore-conjugated secondary antibody for 1 hour and mounted in medium containing DAPI.

For immunofluorescence staining of frozen kidneys, 6$\mu \mathrm{m}$ sections (Leica CM3050S cryostat; Leica Microsystems, Wetzlar, Germany) were fixed in acetone, blocked with $10 \%$ normal donkey serum, and incubated with the primary antibodies, as indicated, overnight at $4^{\circ} \mathrm{C}$. After rinsing with PBS, sections were incubated with conjugated secondary antibodies for 1 hour at room temperature and mounted in medium containing DAPI. Confocal images were obtained using a Leica Inverted-2 microscope with a $63 \times$ oil immersion objective.

\section{Western Blot Analysis}

After 10 to 14 days of differentiation, cells were lyzed on ice in radioimmunoprecipitation assay buffer $[50 \mathrm{mmol} / \mathrm{L}$ Tris (pH 7.5), $150 \mathrm{mmol} / \mathrm{L} \mathrm{NaCl} 0.5 \%$ sodium deoxycholate, $1 \%$ nonidet $\mathrm{P}-40$, and $0.1 \%$ SDS] supplemented with protease inhibitors (cOmplete, Mini; Roche Diagnostics), $1 \mathrm{mmol} / \mathrm{L}$ sodium orthovanadate, $50 \mathrm{mmol} / \mathrm{L} \mathrm{NaF}$, and $200 \mu \mathrm{g} / \mathrm{L}$ okadaic acid. The lysates were centrifuged for 15 minutes at $12,000 \mathrm{rpm}$ and $4^{\circ} \mathrm{C}$. Aliquots of the supernatants $(10 \mu \mathrm{g}$ protein per lane) were separated via $10 \%$ or $12 \%$ SDSPAGE and transferred to polyvinylidene difluoride membrane (Immobilon-P; Millipore). Protein was quantified using the BCA Protein Assay Kit (Pierce Chemical Co., Thermo Fisher Scientific, Inc., Rockford, IL). After incubation with primary and horseradish peroxidase-labeled secondary antibody, the complexes were visualized using SuperSignal West Pico Chemiluminescent Substrate (Pierce Chemical Co.) according to the manufacturer's protocol.

\section{Subcellular Fractionation}

For subcellular fractionation, differentiated podocytes were harvested using trypsin-EDTA, and the various cell fractions were isolated using the Subcellular Protein Fractionation Kit (Pierce Chemical Co.) according to the protocol recommended by the manufacturer. The fractions of soluble nuclear extract and chromatin-bound nuclear extract were pooled to achieve a generic nuclear fraction. 
Preparation of Plasma Membranes

Plasma membranes were separated using the Membrane Protein Extraction Kit (PromoCell $\mathrm{GmbH}$, Heidelberg, Germany) according to the protocol recommended by the manufacturer. The plasma membrane fraction was dissolved in $0.5 \%$ Triton X-100 in PBS. Protein was quantified using Precision Red Protein Assay Reagent (Cytoskeleton) and 10 $\mu \mathrm{g}$ of each preparation separated via $10 \%$ SDS-PAGE.

\section{Cell Size Measurements and Quantification of Focal Adhesions}

Cell size measurements and quantification of focal adhesions were performed using ImageJ software (version $1.44 \mathrm{p}$ ), as described by Marg et al. ${ }^{28}$ On the basis of antivinculin stainings, grayscale 16-bit images were obtained using a Leica DMLB microscope ( $\times 40$ magnification) and processed using ImageJ software. With the use of several commands, the program defines the cell edge and measures the cell area. By using the "thresholding" tool to define the contacts and with the command "Subtract background," the program creates a binary footprint of the adhesion sites. To measure the number of focal adhesions all particles $>0.1$ $\mu \mathrm{m}^{2}$ were counted using the "Analyze particles" command.

\section{Activity Assays}

For activity measurements of the small GTPases, we used Rac1, Rho, and Cdc42 Activation Assay Biochem Kits (Cytoskeleton). For each assay, $700 \mu \mathrm{g}$ protein lysate was incubated with PAK-PBD protein beads (Rac1 and Cdc42) or Rhotekin-RBD beads (RhoA) for 1 hour at $4^{\circ} \mathrm{C}$. After washing, bound proteins were separated via 12\% SDSPAGE and blotted on polyvinylidine difluoride membranes. After incubation with antibodies specific for Rac1, RhoA, and $\mathrm{Cdc} 42$ and the appropriate secondary antibodies, membranes were developed using SuperSignal West Pico Chemiluminescent Substrate (Pierce Chemical Co.) according to the manufacturer's protocol. For determination of total amounts of RhoA, Rac1, and Cdc42, $50 \mu \mathrm{g}$ total protein lysate was used for Western blotting.

\section{Real-Time PCR}

Total RNA was prepared using the RNeasy Mini Kit (Qiagen $\mathrm{GmbH}$, Hilden, Germany) following the protocol recommended by the manufacturer with an additional step of DNase digestion (RNase-Free DNase Set; Qiagen). Total RNA, $1 \mu \mathrm{g}$, was reverse transcribed using Oligo(dT) 15 and random primers and Moloney Murine Leukemia Virus Reverse Transcriptase (Promega GmbH, Mannheim, Germany). Real-time PCR was performed using a Light Cycler 480 (Roche Diagnostics). cDNA was amplified using Fast Start Taq Polymerase (Roche Diagnostics), SYBR Green (Invitrogen), gene-specific primers, and the following PCR conditions: 5 minutes at $95^{\circ} \mathrm{C}$ for 45 cycles, and 10 seconds at $95^{\circ} \mathrm{C}, 10$ seconds at $60^{\circ} \mathrm{C}$, and 10 seconds at $72^{\circ} \mathrm{C}$. Specificity of the amplification product was verified via melting curve analysis.

The samples were measured as multiplexed reactions and normalized to the constitutive gene mouse hypoxanthine phosphoribosyltransferase 1 .

All primers for the listed transcripts were designed using Primer3 software: PKCı: 5'-AGGAACGATTGGGTTGTCAC-3'， 5'-GGCAAGCAGAATCAGACACA-3'; PKC 5'-GCCTCCCTTCCAGCCCCAGA-3'， 5'-CACGGACTCCTCAGCAGACAGCA-3'; Def-6: 5'-CACCAACGTGAAACACTGGA-3', 5'-TGGTGGTGGGTCGCTTAT-3'; and HPRT-1: 5'-CAGTCCCAGCGTCGTGATTA-3', 5'-AGCAAGTCTTTCAGTCCTGTC-3'

\section{Microarray-Based mRNA Expression Analysis (Single- Color Mode)}

The Whole Mouse Genome Oligo Microarray (G4122F, ID 014868; Agilent Technologies, Inc., Santa Clara, CA) used in the present study contains 45,018 oligonucleotide probes covering the entire murine transcriptome. Synthesis of Cy3labeled cRNA was performed using the Quick Amp Labeling kit, one color (No. 5190-0442; Agilent Technologies) according to the manufacturer's recommendations. cRNA fragmentation, hybridization, and washing steps were also performed exactly as recommended, using the One-color Microarray-Based Gene Expression Analysis Protocol version 5.7. Slides were scanned using the Agilent Micro Array Scanner G2565CA at two different photomultiplier tube settings (100\% and 5\%) to increase the dynamic range of the measurements (extended dynamic range mode). Data extraction was performed using Feature Extraction Software version 10.7.1.1 by using the recommended default extraction protocol file: GE1_107_sep09.xml. Processed intensity values of the green channel (gProcessedSignal, or gPS) were normalized via global linear scaling: All gPS values of one sample were multiplied by an array-specific factor. This scaling factor was calculated by dividing a reference 75 th percentile value (set as 1500 for the entire series) by the 75th percentile value of the particular microarray (Array i in the formula below). Accordingly, normalized gPS values for all samples (microarray data sets) were calculated using the following formula: Normalized $\mathrm{gPS}_{\text {Array } \mathrm{i}}=\mathrm{gPS}_{\text {Array } \mathrm{i}} \times$ (75th Percentile Reference Array $/ 75$ th Percentile Array $_{\mathrm{i}}$ ).

A lower intensity threshold was defined as $1 \%$ of the reference 75 th percentile value (threshold $=15$ ). All of those normalized gPS values that fell below this intensity border were substituted for by the respective surrogate value of 15. Calculation of ratio values of relative gene expression was performed using Excel macros.

Heat maps were generated using the MultiExperiment Viewer 4.5.1 program. The intensity values of all genes were logarithmized, and the mean intensity value of each single gene was subtracted from all values. These data were 
loaded into the MultiExperiment Viewer and clustered using the command "Hierarchical clustering" and the adjustments "Euclidean distance" and "Average linkage clustering."

\section{Quantification of Def-6 Expression in Glomeruli}

For quantification of Def-6 expression, cryosections of wildtype (WT) and knockout kidneys were double stained with nidogen and Def-6. Def-6 expression in the podocytic area of glomeruli was rated using a semiquantitative score $(0=$ none, $1=$ less, $2=$ median, $3=$ enhanced, $4=$ strong) by a scientist blinded to the study (M.S.). From each genotype, 40 glomeruli from four animals were scored. The podocytic area was determined by subtraction of the mesangial area marked by nidogen and the Def- 6 -positive area in the glomeruli.

\section{Statistical Analysis}

Data are given as means \pm SD and were compared using unpaired Student's $t$-tests. Data analysis was performed using Excel statistical software. Significant differences were accepted at $P<0.05$.

\section{Results}

Isolation and Characterization of $P K C \lambda / l-$ and $P K C \zeta$ Deficient Podocytes

In a previous study, we identified $\mathrm{PKC} \lambda / \mathrm{\iota}$ as a critical factor for podocyte polarity and architecture. ${ }^{5}$ To generate a PKC $\lambda$ / ı-deficient podocyte cell line, we crossed PKC $\lambda /$ flox/flox Podocin-Cre mice with Immorto transgenic animals expressing the temperature-sensitive SV40 large $\mathrm{T}$ antigen (tsA58TAg) under control of the $\gamma$-interferon-inducible $\mathrm{H}$ $2 \mathrm{~K}^{\mathrm{b}}$ promoter. ${ }^{25}$ Glomeruli were extracted from deficient and control mice, outgrowing cells were isolated, and cell lines were cultured from single cells. To compare the two atypical $\mathrm{PKC}$ isoforms $\mathrm{PKC} \lambda / \mathrm{t}$ and $\mathrm{PKC} \zeta$ in cell culture experiments, we also generated monoclonal cell lines from mice deficient in PKC $\zeta^{6}$ and control mice of the same strain. Three cell lines of each genotype were characterized using PCR analysis and various podocyte-specific antibodies (Supplemental Figure S1). After differentiation, podocyte marker proteins synaptopodin ${ }^{29}$ and WT- $1^{30}$ were detected in all cell lines via immunofluorescence staining, and expression of nephrin, podocin, and WT-1 were confirmed in cell lysates via Western blot analysis (data not shown).

\section{PKC $\lambda /$ ı Deficiency Influences the Actin Cytoskeleton and Motility of Podocytes}

Under permissive conditions at $33^{\circ} \mathrm{C}$, WT podocytes usually grow in a confluent cobblestone-like pattern. After 10 to 14 days of differentiation, podocytes spread out and show an arborized shape. ${ }^{27}$ We observed this expected appearance under permissive $\left(33^{\circ} \mathrm{C}\right)$ and nonpermissive $\left(37^{\circ} \mathrm{C}\right)$ conditions in WT but not in $P K C \mathrm{~N}^{-1-}$ podocytes. $P K C \mathrm{~N}^{-1-}$ podocytes exhibited a more spindle-like shape and grew in clusters, never reaching confluence at $33^{\circ} \mathrm{C}$ (data not shown). After differentiation at $37^{\circ} \mathrm{C}, P K C \mathrm{~N}^{-1-}$ podocytes were notably smaller and possessed longer cell protrusions than did control cells. $P K C \zeta^{-1-}$ podocytes showed a phenotype similar to that of WT cells (Figure 1A). Live cell imaging was used to record the cell movement of WT and deficient cells for 6 hours, with an image obtained every 2 minutes. We measured the distance moved for 10 cells per genotype (Figure 1B) by marking and following the cell nucleus in the image sequence using ImageJ software. $P \mathrm{KCN}^{-1-}$ podocytes moved a substantially longer distance within 6 hours of measurement in comparison with WT cells. The distance moved by $\mathrm{PKC}^{-1-}$ podocytes was similar to that of WT cells (Figure 1C). We hypothesized that the irregular cell shape and altered movement of $P K C \mathrm{Ni}^{-1-}$ podocytes was due to a disturbed actin cytoskeleton. Staining of PKC $\mathrm{N}_{\mathrm{L}}$ deficient and WT podocytes with phalloidin to visualize actin revealed a substantially altered cytoskeleton with fewer stress fibers and less accumulation of actin fibers at the cellular edges in $74 \%$ of the PKC $\lambda / \mathrm{t}$-deficient cells (Figure 1D) (10 high-power fields, $\times 20$ magnification) containing 10 to 40 cells were analyzed from each genotype. Cells were characterized as having a "rearranged actin cytoskeleton" when they did not exhibit regular stress fiber patterns but an actin ringlike structure with actin accumulation at cellular edges. In contrast, the percentage of cells with a rearranged cytoskeleton in $P K C \zeta^{-1-}$ podocytes $(16 \%)$ was comparable to that in WT cells $(23 \%)$. In agreement with these findings, treatment of WT cells with PKC $\lambda / \mathrm{t}$ pseudosubstrate and highly effective PKC $\lambda / \iota$ siRNA (approximately 95\%; Figure $1 \mathrm{~F}$ ) resulted in changes to the actin cytoskeleton similar to those observed in $P K C \mathrm{~N}^{-1-}$ podocytes, indicating a role for PKC $\lambda / \mathrm{s}$ but not for $\mathrm{PKC} \zeta$ in actin regulation and stress fiber formation in vitro (Figure 1E).

\section{PKC $\lambda /$ ı Deficiency Leads to Increased Activation of Small GTPases, Predominantly Rac1}

Formation and motility of the actin cytoskeleton is regulated by a group of small GTPases. ${ }^{31}$ Small GTPases can exist in inactive GDP-bound or active GTP-bound forms. Activation of small GTPases is regulated by GEFs that trigger the exchange rate of GDP and GTP. ${ }^{15}$ GTPaseactivating proteins lead to inactivation of small GTPases. ${ }^{32}$ The Rho GTPases (Rho, Rac, and Cdc42) control signaling pathways for assembly and disassembly of the actin cystoskeleton and associated integrin adhesion complexes. ${ }^{33}$ Activation levels of small Rho GTPases RhoA, Rac1, and Cdc42 were determined using pull-down assays, and were normalized to total levels of each respective protein (Figure 2A). Furthermore, the values of the deficient cell lines were normalized to the appropriate WT cell line. Normalization revealed significantly increased levels 
A
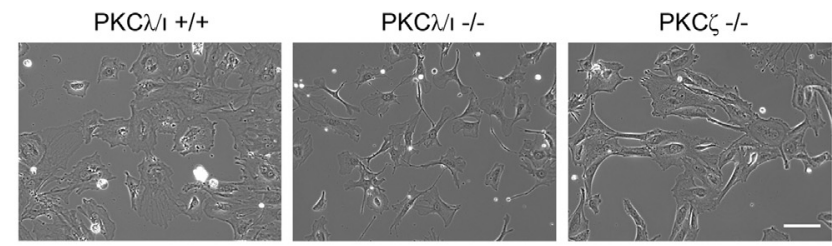

B
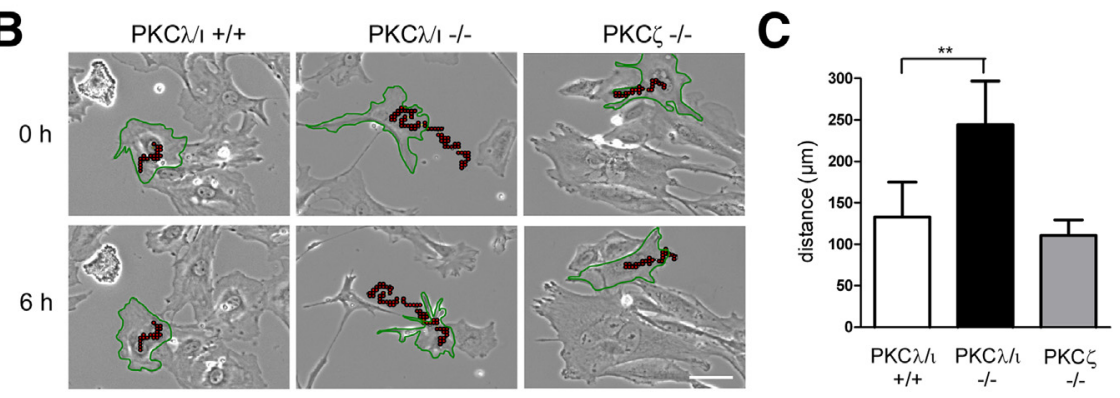

D
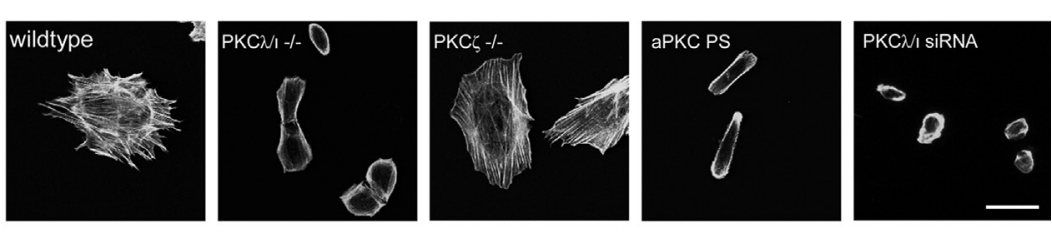

$\mathbf{E}$

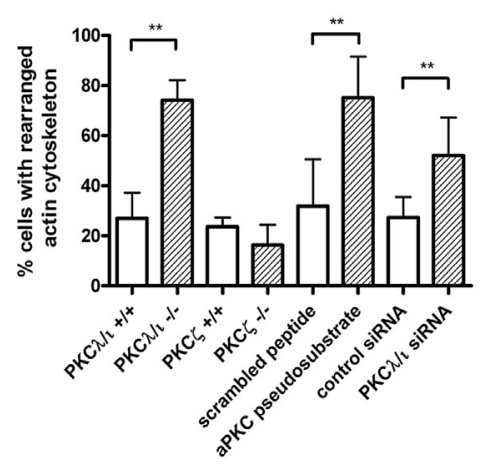

$\mathbf{F}$

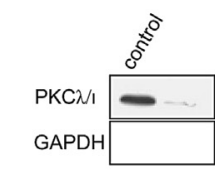

Figure 1 Phenotype of $P K C \lambda / \mathrm{l}$ - and $P K C \zeta$ deficient podocytes in vitro. A: Phenotype of deficient or control podocytes after 10 days of differentiation at $37^{\circ} \mathrm{C}$. Images of live cells were obtained using a light microscope (Axio Observer.Z1, $\times 20$ magnification). $P K C \mathrm{~N} / \mathrm{i}^{-/-}$podocytes grow spindle-like and do not reach confluence, in contrast to control and $\mathrm{PKC}^{-/-}$cells. Scale bar $=$ $100 \mu \mathrm{m}$ (applies to all panels). B: Migration of deficient and control podocytes recorded using a live microscope (Axio Observer.Z1, $\times 20$ magnification). The observed cells are shown in green, and the covered distance is illustrated as red dots. Scale bar $=50 \mu \mathrm{m}$ (applies to all panels). C: Distance covered from startup to 6 hours, measured using ImageJ software (Manual Tracking), was significantly increased in $P K C \mathrm{I}^{-/-}$ podocytes. $n=10$ cells per genotype. D: Phalloidin staining reveals increased number of cells with rearranged cytoskeleton in $P K C \mathrm{~N}_{\mathrm{i}}{ }^{-1-}$ podocytes, podocytes treated with aPKC pseudosubstrate (PS) or with $P K C \lambda / \mathrm{l}$ siRNA, in comparison with WT podocytes. $P K C \zeta^{-/-}$podocytes showed no difference from WT cells. Scale bar $=50 \mu \mathrm{m}$ (applies to all panels). E: Quantification of cells with rearranged cytoskeleton. Each treated cell line was compared with an appropriate control. Ten highpower fields ( $\times 20$ magnification) containing 10 to 40 cells were analyzed from each genotype using a Leica DMBL microscope. F: Transfection with $P K C \lambda / \mathrm{l}$ siRNA significantly reduced the amount of $\mathrm{PKC} \lambda / \mathrm{l}$ in WT podocytes. ${ }^{*} P<0.01$. of activated RhoA and $\mathrm{Cdc} 42$ and a large increase in activated levels of Rac1 in $P \mathrm{KCN}^{-/-}$podocytes. Rac1 activity in $P \mathrm{KC} \zeta^{-/-}$podocytes was also slightly increased, but to a much lesser degree than in $P \mathrm{KCN}^{-1-}$ podocytes. In addition, in both deficient cell lines, total RhoA was considerably reduced, whereas total Rac1 and total Cdc42 were only slightly affected. Equal sample loading was confirmed by testing the lysates for the expression levels of GAPDH. Quantification of signal intensity from four experiments in three independent cell clones confirmed these results (Figure 2B). These data indicate a regulatory effect of $\mathrm{PKC} \lambda / \mathrm{s}$ on all tested small GTPases, with a predominance for Rac1.

\section{PKC $\lambda /$ I Deficiency Influences Cell Size and Number of Focal Adhesions of Podocytes}

The focal adhesion complex can influence the actin cytoskeleton, and vice versa, via direct connection of stress fibers to this multiprotein complex. The primary components of focal adhesions that link the extracellular matrix to the cytoskeleton are vinculin and integrins. ${ }^{34}$ Vinculin stainings of differentiated podocytes revealed differences in the distribution pattern and number of focal adhesions in $\mathrm{PKC} / \mathrm{t}$ deficient cells compared with WT control cells (Figure 3A). Twenty high power fields ( $\times 40$ magnification) of each genotype were analyzed, and the experiments were performed three times in different cell clones. The total cell area was significantly reduced in $P \mathrm{KCN}^{-/-}$podocytes (Figure $3 \mathrm{~B}$ ), whereas the cell area of $P K C \zeta^{-/-}$podocytes was significantly increased in comparison with WT cells (Figure 3C). In addition, the number of focal adhesions was significantly reduced in $\mathrm{PKCN}^{-/-}$podocytes, whereas the number of focal adhesions in $P K C \zeta^{-/-}$cells was unaffected. Overall, the size of the focal adhesions was similar among deficient cell lines and WT cells; however, analysis of the number of adhesions relative to $100-\mu \mathrm{m}^{2}$ cell area revealed a significant increase in the density of adhesions in $P \mathrm{KCN}^{-1-}$ podocytes 
A

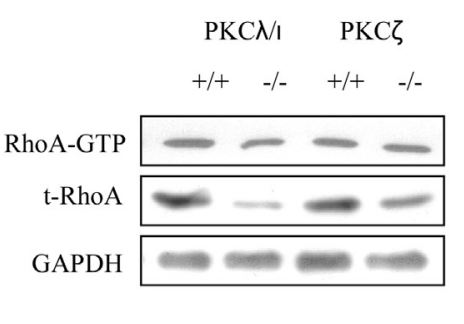

B

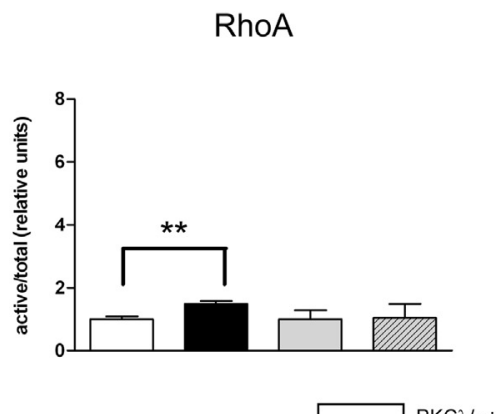

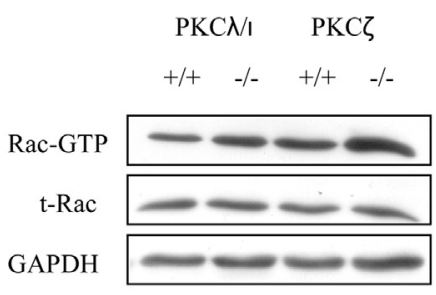

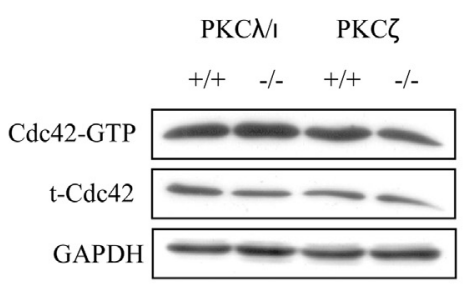

Cdc42
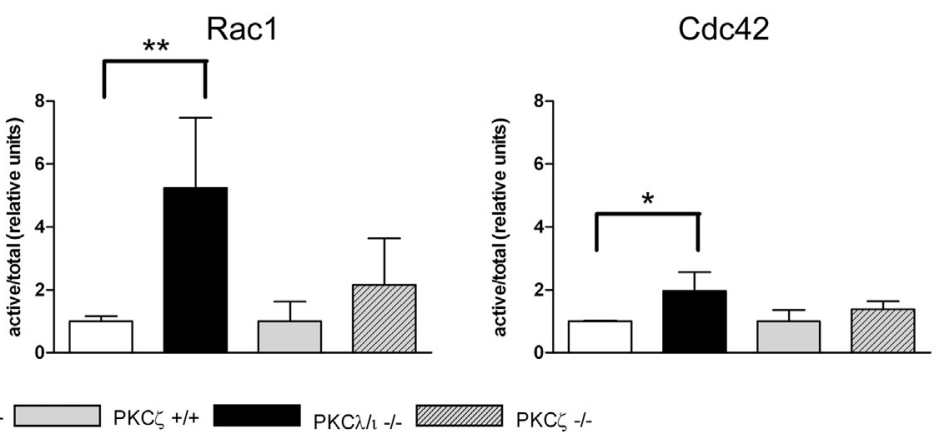

Figure $2 \mathrm{PKC} \lambda / \mathrm{l}$ deficiency influences activation of small GTPases. A: Protein lysates of deficient and control podocytes were used in activity assays for the small GTPases RhoA, Rac1, and Cdc42 and were analyzed using Western blot analysis. For loading control, the blotted membranes were incubated with GAPDH. B: Quantification of active GTPase versus total GTPase expression levels of RhoA, Rac1, and Cdc42. At least three different experiments of independent cell clones were used for each quantification. ${ }^{*} P<0.05,{ }^{* *} P<0.01$.

and decreased density in $P K C \zeta^{-/-}$cells. Similarly, we detected an influence on the relative adhesion area by building the ratio of adhesion area to whole cell area (Figure 3, B and C). This analysis revealed that although the number of focal adhesions was significantly reduced in $P K C \mathrm{Nl}^{-/-}$cells, the reduced cell area leads to an increased density of adhesions and relative adhesion area. This result was confirmed in various clones from each genotype. This clearly indicates that the primary effect influencing the phenotype is reduced cell spreading and not the number of focal adhesions. Western blot analysis of integrin $\beta 1$ strengthened evidence for an influence of PKC $\lambda / \iota$ deficiency on the focal adhesion complexes. Integrin $\beta 1$ expression was significantly decreased in $P K C \mathrm{~N}^{-1-}$ podocytes but was not significantly affected in $P K C \zeta^{-/-}$podocytes (Figure 3D). Although the small increase in integrin $\beta 1$ in $P K C \zeta^{-1-}$ podocytes was not significant in comparison with $P K C \zeta^{+/+}$ or $P K C \mathrm{~N}_{\mathrm{i}}^{+/+}$podocytes, it is an interesting finding. The common connection of integrin and aPKCs in migration and cell polarity ${ }^{35}$ could represent counterregulation of the aPKC isoforms $\zeta$ and $\lambda / \mathrm{t}$ (Figure 3E). The expression of vinculin was decreased in both deficiencies; however, densitometric analysis of three independent experiments in various cell clones indicated no statistical significance (Figure 3E).

\section{Def- 6 mRNA Is Increased in PKC $\lambda / \mathrm{l}^{-/-}$Podocytes}

To identify downstream targets of aPKCs that potentially regulate the actin cytoskeleton in podocytes, we performed a comparative microarray analysis of $a P K C^{-/-}$and WT podocytes. For all array experiments, mRNA samples from two independently generated cell lines were used per genotype to exclude clonal effects. The lists of differentially expressed genes were sorted, and only the genes more than twofold up-regulated or down-regulated in both cell lines were used in the final analysis. The resulting lists of upregulated or down-regulated genes (Supplemental Tables S1 through S4) in aPKC-deficient cell lines revealed 67 common genes that are more than twofold regulated in both aPKC isoform deficiencies, whereas 297 genes were differentially regulated in only $P K C \zeta^{-1-}$ cells, and 593 genes were differentially regulated in only $P \mathrm{KCN}^{-1-}$ cells (Figure 4).

On the basis of the discrepancy between the phenotypes of $\mathrm{PKC} \lambda / \mathrm{l}$ and $\mathrm{PKC} \zeta$ deficiency, we focused on genes being changed in their expression in PKC $/ \mathrm{t}$ - but not $\mathrm{PKC} \zeta$-deficient cells. The top 30 up-regulated and down-regulated genes regulated only in $P K C \mathrm{~N}_{\mathrm{i}}^{-/-}$podocytes were clustered in heat maps using the MultiExperiment Viewer program (Figure 4). This analysis revealed that several genes exclusively regulated in $P K C \mathrm{~N}^{-1-}$ podocytes but not in $P K C \zeta^{-/-}$ cells (Tables 1 and 2) were changed more than 100-fold. Gene ontology analysis performed using Onto-Express revealed that a substantial number of genes involved in cytoskeletal adaptor activity, G-protein coupled receptor activity, cell adhesion, and filopodium formation were affected (Supplemental Table S5). Furthermore, Pathway-Express analysis detected the cell adhesion molecules as one of the significantly changed pathways in $P K C \mathrm{~N}^{-1-}$ but not $P K C \zeta^{-/-}$podocytes (Supplemental Tables S6 through S8).

Def-6, a $74-\mathrm{kDa} G E F,{ }^{21}$ was one of the strongest regulated genes in $P K C \mathrm{~N}^{-1-}$ podocytes (24-fold up-regulated). 
A
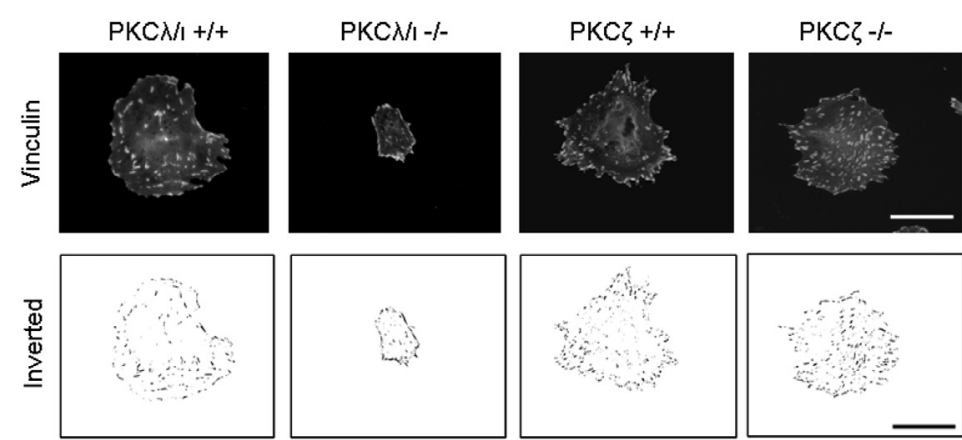

cell area

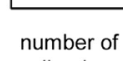

adhesions

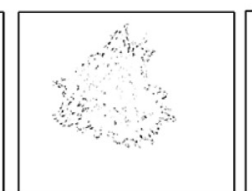

$\begin{array}{ll}\text { size of } & \text { density of } \\ \text { adhesions } & \text { adhesions }\end{array}$

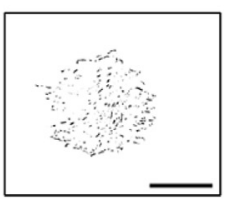

adhesions adhesion area

B
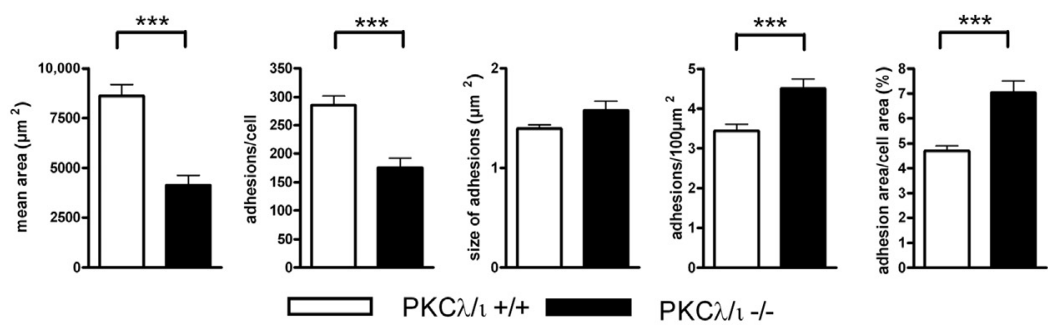

C
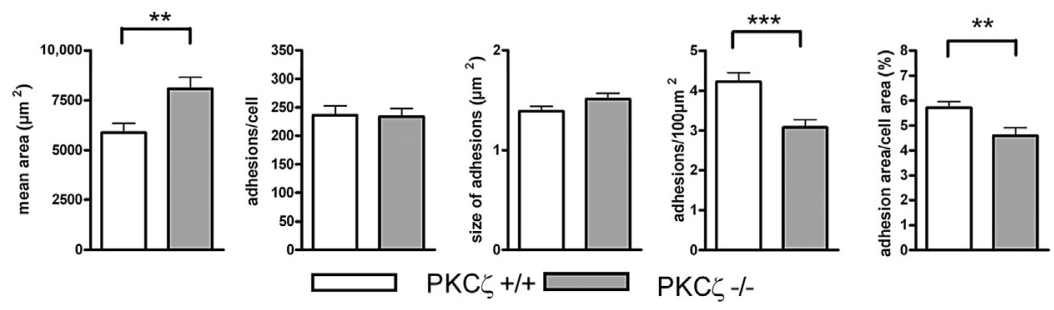

D

E

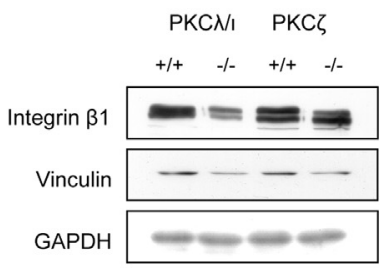

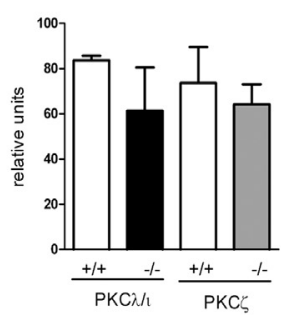

Figure $3 \quad P K C \lambda / \iota$ deficiency influences the cell size and adhesion molecules of podocytes. A: Deficient and control podocytes were stained with an antibody against vinculin, and grayscale images were obtained using a Leica DMBL microscope $(\times 40$ magnification). Binary inverted images were constructed using ImageJ macros to obtain footprints of vinculin distribution. Measurement of cell area and number and size of focal adhesions was performed using ImageJ for PKC $\lambda /$ I-deficient cells (B) and PKC -deficient cells (C) compared with WT cells. Twenty high-power fields ( $\times 40$ magnification) of each genotype were analyzed, and the experiments were performed three times in different cell clones. Scale bar $=50 \mu \mathrm{m}$ (applies to all panels). D: Western blot analysis reveals significantly reduced levels of integrin $\beta 1$ in $P K C N / \mathrm{i}^{-/-}$podocytes and a nonsignificant reduction in vinculin in $P K C \mathrm{~N}_{\mathrm{i}}{ }^{-1-}$ and $P K C \zeta^{-1-}$ podocytes. Lysates were normalized to GAPDH. E: Quantification of at least three independent experiments of different cell clones. ${ }^{*} P<$ $0.05,{ }^{*} p<0.01$, and ${ }^{* * *} P<0.001$.
Def-6 has been analyzed in various cell types; however, to date, no functional studies of renal cells have been published. Western blot experiments and real-time PCR measurements were performed to confirm the results of the microarrays. Western blot analysis clearly demonstrated the absence of $\mathrm{PKC} \lambda / \mathrm{s}$ protein in $\mathrm{PKC} \lambda / \mathrm{t}$-deficient podocytes and the absence of $\mathrm{PKC} \zeta$ protein in $\mathrm{PKC} \zeta$-deficient cells. mRNA levels of $\mathrm{PKC} \lambda / \mathrm{\imath}$ and $\mathrm{PKC} \zeta$ were significantly down-regulated in the appropriate deficient cell lines. Total Def-6 protein levels were comparable in WT and both deficient cell lines; however, as detected in the microarray, the mRNA of Def-6 was significantly upregulated in $P \mathrm{KCN}^{-/-}$podocytes. In $P K C \zeta^{-/-}$cells, there was no detectable changes in WT cells (Supplemental Figure S2).

\section{Def-6 Expression in Glomeruli and Localization in Podocytes}

Def-6 is highly expressed in lymphatic organs such as spleen and thymus. ${ }^{21}$ Def- 6 could also be detected in various muscle tissues and in renal HEK293 cells. ${ }^{23}$ Inasmuch as currently nothing is known about Def-6 in the kidney, it was of great interest to examine in which renal compartments Def-6 is expressed. Therefore, we stained $P K C \mathrm{Ni}^{+/+}$and $\mathrm{PKC \textrm {N } ^ { - / - }}$ kidney cryosections with an 

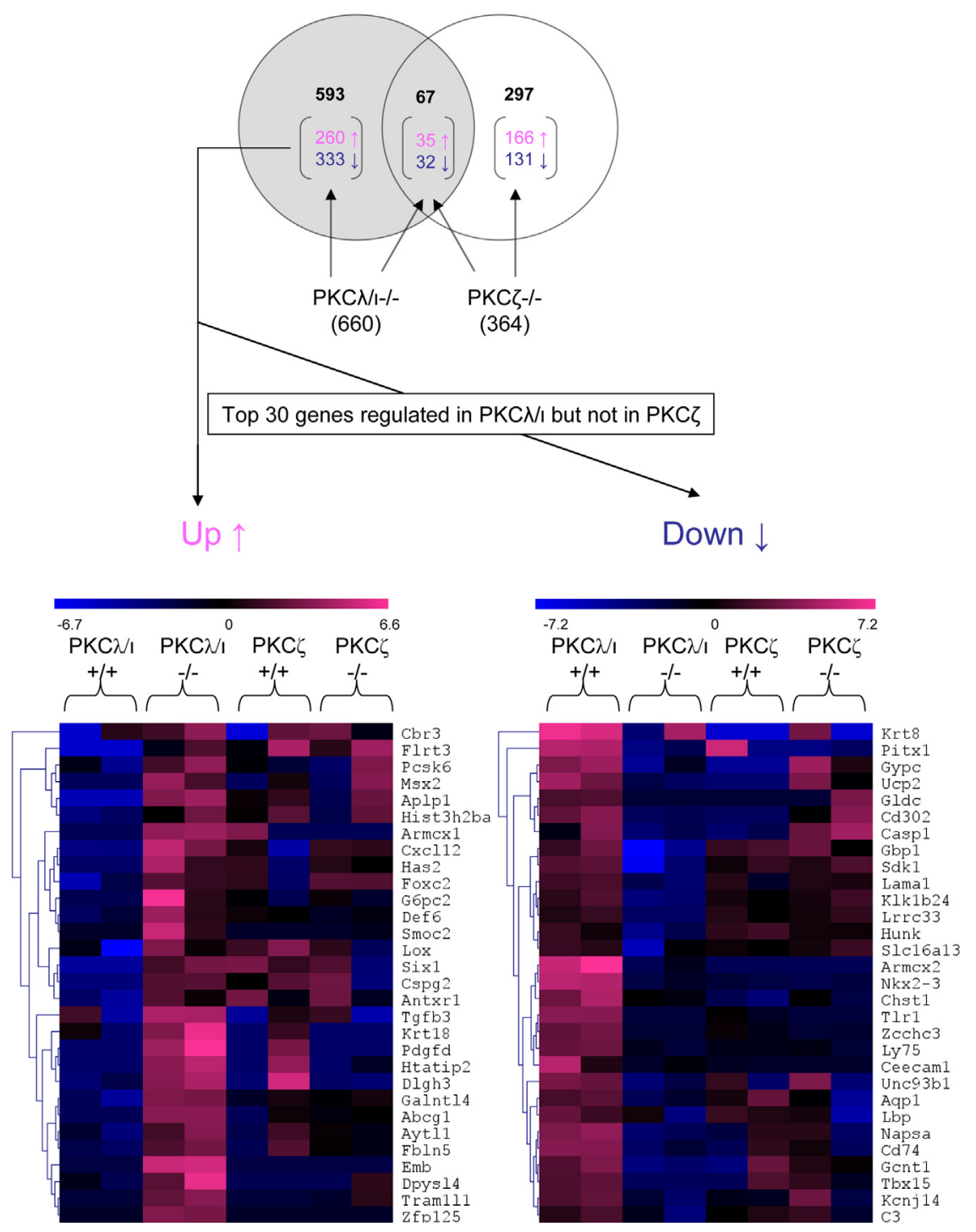

Figure 4 Microarray analysis of $\mathrm{PKC} \lambda / \mathrm{L}$ - and PKC $\zeta$-deficient podocytes. Diagram shows the total number of regulated genes in cells deficient for PKC $\lambda / \mathrm{\iota}$ or PKC $\zeta$ and the number of more than twofold up-regulated $\uparrow$ or more than twofold down-regulated $\downarrow$ genes (in brackets) and the intersection of the two compared conditions. The top 30 genes up-regulated or down-regulated in PKC $\lambda / \mathrm{l}$ - but not in PKC -deficient cells are illustrated in heat maps. Intensity of regulation is shown as low expression (blue) or high expression (pink). Genes are clustered according to similar intensity using the Software Multi Experiment Viewer at the left side of each heat map. For each genotype, two different cell lines were analyzed in the microarray. antibody against Def-6. The staining demonstrated that in the kidney Def-6 is predominantly expressed in the glomeruli and is only weakly expressed in the tubules (Figure 5A). Furthermore, to test the specificity of the Def-6 antibody, we preincubated the antibody for 45 minutes with equal concentrations of Def-6 blocking peptide (Def6 recombinant protein); both secondary antibody and antibody blocking control were normal (Figure 5A). Co-staining with the podocyte-specific marker podocalyxin and Def-6 demonstrated that Def-6 is expressed in podocytes (Figure 5B). Semiquantitative scoring analysis of Def-6 expression in glomeruli of WT and $P K C \mathrm{~N}^{-1-}$ mice $(n=$ 4 mice of each genotype, $\geq 10$ glomeruli per mouse) showed that Def-6 protein expression is significantly higher in podocytes of $\mathrm{PKCN}^{-/-}$glomeruli than of WT mice (Figure 5C). The medium score of Def-6 expression in the podocytic area of $P K C \mathrm{~N}^{-/-}$glomeruli was significantly higher in comparison with WT glomeruli (3.2 versus 2.0) (Figure 5D). The changed score distribution of $\mathrm{PKCN} \mathrm{i}^{+/+}$ and $P \mathrm{KCN}^{-1-}$ glomeruli is shown in Figure 5D. To investigate the expression of Def-6 in vitro, we co-stained differentiated WT and $P K C \mathrm{Ni}^{-1-}$ podocytes with antibodies against Def-6 and vinculin to mark focal contacts. We detected that endogenous Def-6 localized to the cellular edges and co-localized with vinculin at focal adhesions in podocytes. Furthermore, $P K C \mathrm{Ni}^{-/}$cells had more pronounced membrane-associated expression of Def-6 (Figure 5E). Subcellular fractionation analysis of protein lysates revealed that Def-6 is primarily expressed in the podocyte membrane and only negligibly in the cytosolic or nuclear fraction. The membrane-associated expression is significantly enhanced in $P K C \mathrm{~N}^{-1-}$ podocytes $(140 \%$ in comparison with the WT membrane fraction), which was confirmed via quantification of fractionations from three independent experiments of different cell clones (Figure 5F). Moreover, in specific preparations of isolated plasma membranes of $P K C \mathrm{Ni}^{+/+}$and $P K C \mathrm{~N}^{-1-}$ podocytes, we could specify the enhanced membrane localization 
Table 1 Highest up-regulated genes in $P K C \mathrm{~N}_{\mathrm{i}}^{-/-}$podocytes in comparison with WT, which are not regulated in $\mathrm{PKC \zeta}^{-/-}$ podocytes

\begin{tabular}{|c|c|c|}
\hline Gene & Systematic Name & Fold Change \\
\hline Aplp1 & NM_007467 & 367.78 \\
\hline Pdgfd & BC030896 & 269.44 \\
\hline Cxcl12 & NM_013655 & 166.29 \\
\hline$E m b$ & NM_010330 & 134.34 \\
\hline Htatip2 & NM_016865 & 126.05 \\
\hline Six1 & NM_009189 & 86.55 \\
\hline Dlgh3 & NM_016747 & 86.51 \\
\hline Galntl4 & NM_173739 & 81.09 \\
\hline Armc $x 1$ & NM_030066 & 71.40 \\
\hline Flrt3 & NM_178382 & 64.97 \\
\hline Krt18 & NM_010664 & 60.59 \\
\hline$A b c g 1$ & NM_009593 & 59.72 \\
\hline G6pc2 & NM_021331 & 58.98 \\
\hline Tgfb3 & NM_009368 & 50.17 \\
\hline Dpysl4 & NM_011993 & 49.01 \\
\hline Has2 & NM_008216 & 48.59 \\
\hline Lox & NM_010728 & 43.37 \\
\hline Cspg2 & D16263 & 41.96 \\
\hline Msx2 & NM_013601 & 39.62 \\
\hline Foxc2 & NM_013519 & 34.89 \\
\hline Pcsk6 & XM_355911 & 30.30 \\
\hline Cbr3 & NM_173047 & 29.14 \\
\hline Aytl1 & NM_173014 & 27.49 \\
\hline Fbln5 & NM_011812 & 27.00 \\
\hline Antxr1 & NM_054041 & 24.53 \\
\hline Def6 & NM_027185 & 23.91 \\
\hline Tram1l1 & NM_146140 & 23.13 \\
\hline Hist3h2ba & NM_030082 & 20.82 \\
\hline Zfp125 & AJ005350 & 19.73 \\
\hline Smoc2 & NM_022315 & 19.22 \\
\hline
\end{tabular}

Fold change is given as the geometric mean for all genes more than twofold up-regulated in both tested cell clones.

of Def-6 in the plasma membrane of $P K C \mathrm{Ni}^{-1-}$ podocytes (three independent experiments performed in different cell clones) (Figure 5G).

\section{Actin Phenotype of PKC $\lambda / \mathrm{I}^{-/-}$Podocytes Is Rescued by Overexpression of $\mathrm{PKC} \lambda / \mathrm{\imath}$}

We examined the effect of PKC $\lambda / \mathrm{t}$ overexpression in PKCN $\mathrm{I}^{-/-}$podocytes on the actin cytoskeletal architecture. Podocytes were transduced with adenoviruses expressing PKCı-GFP or GFP alone as control. At 7 days after infection, RNA was isolated or cells were fixed for immunostaining. Because there were no detectable differences in the presence of collagen (data not shown), all experiments were performed in the absence of collagen. Real-time PCR analysis revealed partial rescue of $\mathrm{PKC} \mathrm{I}$ mRNA expression $(60 \%)$ in $\mathrm{PKC} \lambda / \mathrm{t}$-deficient cells after infection with $\mathrm{PKCt}$ GFP (Figure 6A). As a consequence, Def-6 mRNA expression decreased significantly (40\% in comparison with $P K C \mathrm{Nl}^{-/-}$) (Figure 6A). Western blot analysis confirmed
PKCı-GFP or GFP overexpression in WT and deficient cells. Total Def-6 protein expression levels remained unaffected (Figure 6B). Furthermore, phalloidin stainings of rescued $P \mathrm{KCN}^{-1-}$ podocytes revealed normalized actin stress fibers in approximately 50\% of tranfected cells compared with GFP-infected or GFP-uninfected cells (Figure 6C). The experiments were repeated three times in two different cell clones and confirmed these results. Vinculin stainings of transduced podocytes were used to measure the cell area (Figure 6D) and number of focal adhesions (Figure 6D) (20 cells of each genotype, repeated in two different cell clones). Both cell area and number of focal adhesions could be partially rescued after infection of $P K C \mathrm{~N}^{-1-}$ podocytes with PKCl-GFP. The cell area and number of adhesions were significantly increased; however, rescue was detectable in only $50 \%$ in comparison with WT podocytes, which might be due to only partial rescue of $\mathrm{PKC} \mathrm{mRNA}$. Figure $6 \mathrm{E}$ shows representative vinculin stainings after virus transduction. Overexpression of GFPtagged Def-6 FL and constitutively active Def-6, which consists of only the DHL domain, ${ }^{22}$ in WT podocytes

Table 2 Highest down-regulated genes in $P K C \mathrm{~N}_{\mathrm{i}}^{-/-}$podocytes in comparison with WT, which are not regulated in $P K C \zeta^{-/-}$ podocytes

\begin{tabular}{lll}
\hline Gene & Systematic Name & Fold Change \\
\hline Pitx1 & NM_011097 & 262.07 \\
Armcx2 & NM_026139 & 250.64 \\
Gbp1 & NM_010259 & 234.54 \\
Sdk1 & NM_177879 & 168.47 \\
Nkx2-3 & NM_008699 & 104.63 \\
Napsa & NM_008437 & 96.27 \\
Gypc & NM_027863 & 91.91 \\
Gcnt1 & NM_010265 & 69.51 \\
Cd74 & NM_010545 & 63.38 \\
Cd302 & NM_025422 & 53.39 \\
Krt8 & NM_031170 & 52.63 \\
Unc93b1 & NM_019449 & 52.00 \\
Tlr1 & NM_030682 & 45.94 \\
Klk1b24 & NM_010643 & 32.84 \\
Tbx15 & NM_009323 & 32.23 \\
Kcnj14 & NM_145963 & 26.07 \\
Lama1 & NM_008480 & 23.63 \\
Ucp2 & NM_011671 & 22.67 \\
Zcchc3 & NM_175126 & 21.17 \\
Casp1 & NM_009807 & 20.24 \\
Ly75 & AK157921 & 18.66 \\
Chst1 & NM_023850 & 18.26 \\
Aqp1 & NM_007472 & 17.55 \\
Hunk & NM_015755 & 16.81 \\
Slc16a13 & NM_172371 & 16.42 \\
Lrrc33 & NM_146069 \\
Ceecam1 & NM_207298 & 16.33 \\
Lbp & NM_008489 & 14.29 \\
Gldc & NM_138595 & 13.98 \\
C3 & NM_009778 & 13.30 \\
\hline & N & 13.02 \\
\hline
\end{tabular}

Fold change is given as the geometric mean for all genes more than twofold down-regulated in both tested cell clones. 
A

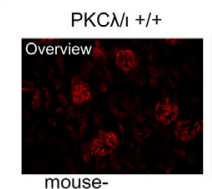

AlexaFluor( 555

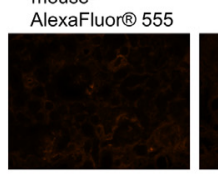

B

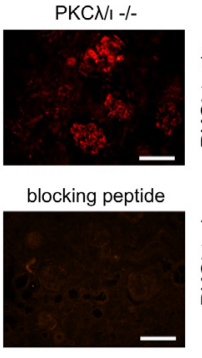

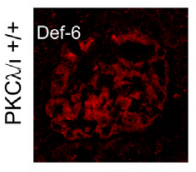

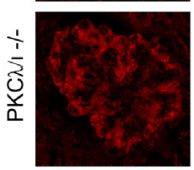

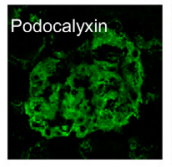
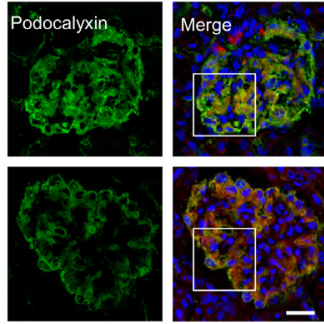

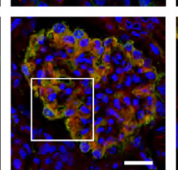

D
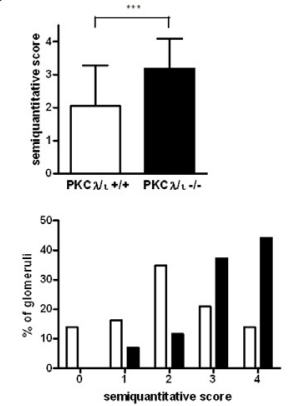

$\mathbf{F}$
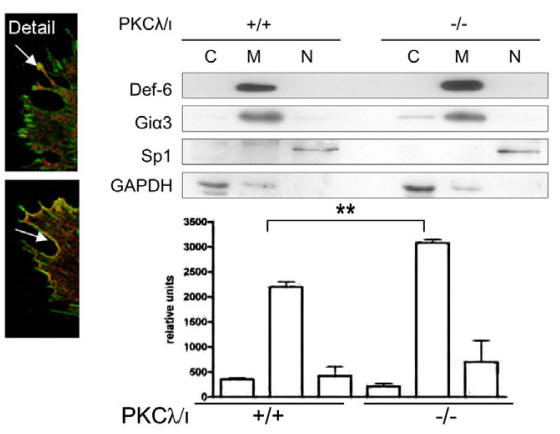

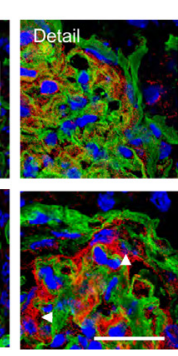

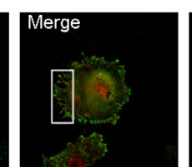
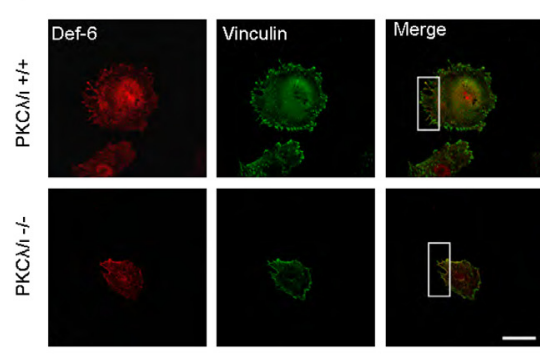

G

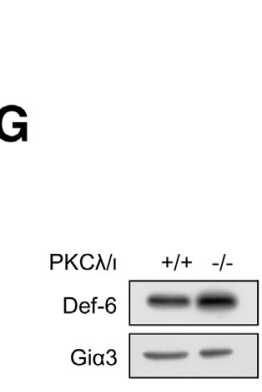

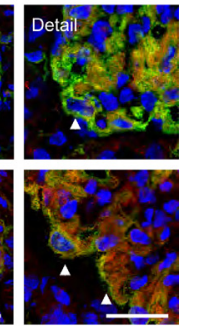

Figure 5 Def-6 expression is enhanced in $\mathrm{PKC} \lambda / \mathrm{t}^{-/-}$glomeruli and localizes to cellular edges in podocytes. A-C: Staining of kidney cryosections from $P K C M / \mathrm{i}^{+/+}$and $P K C \mathrm{~N}_{\mathrm{i}}{ }^{-/-}$mice. A: Upper panels: Overview staining with an antibody against Def-6 ( $\times 10$ magnification) reveals strong glomerular expression. Lower panels: Control staining with secondary antibody or Def-6 blocking peptide validated the specificity of the Def- 6 antibody. Scale bars $=200 \mu \mathrm{m}$. B: Co-staining of Def-6 (red) and the podocyte marker podocalyxin (green) reveals enhanced podocytic expression of Def- 6 in $\mathrm{PKCN} / \mathrm{I}^{-1-}$ glomeruli (boxed areas in Merge views, and arrowheads in Detail views). Nuclei are visualized with DAPI (blue). Scale bars $=25 \mu \mathrm{m}$ (applies to all panels). C: For quantification of Def-6 expression, sections were double-stained against Def-6 (red) and nidogen (green). Nuclei were visualized using DAPI (blue). Def-6 expression is enhanced in the podocytic areas of $P K C M / \mathrm{i}^{-1-}$ glomeruli (boxed areas in Merge views, and arrowheads in Detail views). Scale bars $=25 \mu \mathrm{m}$ (applies to all panels). D: Images of $P K C N / \mathrm{i}^{+/+}$and $P K C N / \mathrm{i}^{-/-}$ glomeruli ( 40 glomeruli from four animals of each genotype) were obtained and analyzed using a semiquantitative score ranging from 0 (no expression) to 4 (strong expression). Compared with WT glomeruli, $P K C \mathrm{~N}_{\mathrm{L}}^{-/-}$glomeruli show a significantly higher score and an obvious change in score distribution. $n=4$ mice of each genotype, $\geq 10$ glomeruli per mouse. ${ }^{* *} P<0.001$ E: Endogenous Def-6 localizes to the cellular edges and co-localizes with vinculin, in particular in the $P K C \mathrm{~N} / \mathrm{I}^{-1-}$ podocytes. Immunostaining of differentiated podocytes with an antibody against Def-6 (red) and vinculin (green) (insets in Merge views, and arrows in Detail views). Scale bar $=50 \mu \mathrm{m}$ (applies to all panels). F: Def-6 membrane localization is significantly enhanced in $P K C \mathrm{I}^{-1-}$ podocytes. Subcellular fractionation of differentiated $P K C \mathrm{~N}_{\mathrm{i}}^{+/+}$and $P K C \mathrm{~N}_{\mathrm{i}}^{-/-}$podocytes. Lysates were normalized with Gi 33 (membrane fraction, M), Sp1 (nuclear fraction, N), and GAPDH (cytosolic fraction, C). Quantitative analysis of Def-6 expression in three independent experiments of two independent cell clones. ${ }^{* *} P<0.01$. G: Isolation of plasma membranes from $P K C N / \mathrm{i}^{+/+}$and $P K C N / \mathrm{i}^{-/-}$ podocytes reveals increased localization of Def- 6 in plasma membranes of $P K C \mathrm{~N}_{\mathrm{i}}^{-1-}$ podocytes in comparison with WT cells on Western blot analysis (left panel). For quantification, lysates of three independent experiments from two independent cell clones were normalized with Gi $\alpha 3$ (right panel). ${ }^{*} P<0.05$.

confirmed membrane localization of active Def-6 in podocytes. In particular, constitutively active Def-6 DHL domain exhibited enhanced Def-6 localization at the cell membrane of the leading edges in transfected podocytes (Figure 6F). Next we performed Def-6 staining on transduced podocytes. After overexpression of PKCı-GFP, the membrane localization of Def-6 in $P K C \mathrm{Ni}^{-/-}$podocytes could be reversed in $>50 \%$ of the cells (Figure $6 \mathrm{G}$ ). Both experiments were performed three times.

In summary, our data suggest that $\mathrm{PKC} \lambda / \mathrm{\iota}$ functions as an upstream regulator of Def-6 activation in podocytes.
Absence of PKC $\lambda / \iota$ leads to an increase in Rac1 activation. In contrast, RhoA and $\mathrm{Cdc} 42$ activation is only mildly affected (Figure 7).

\section{Discussion}

Podocytes are highly specialized, differentiated cells that form and maintain a network of arborized primary and secondary foot processes. The formation of this foot process network is maintained via a tightly orchestrated actin cytoskeleton. The gaps between the secondary foot processes are 
A

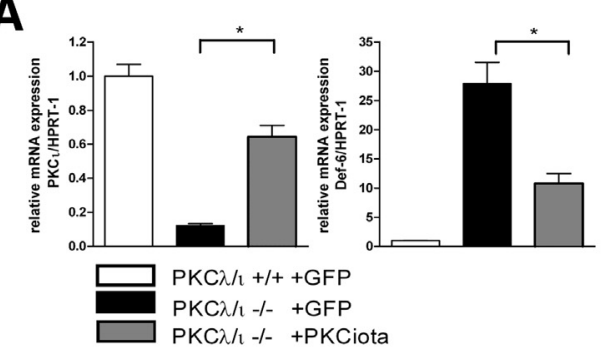

C

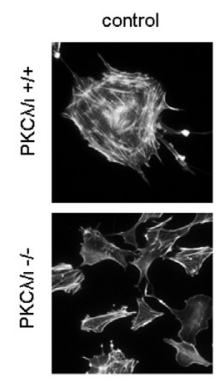

D

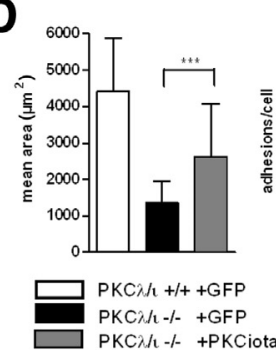

$\mathbf{F}$
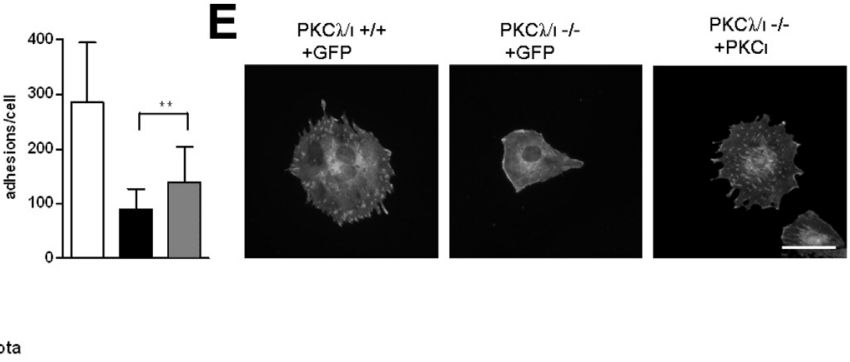

(1)

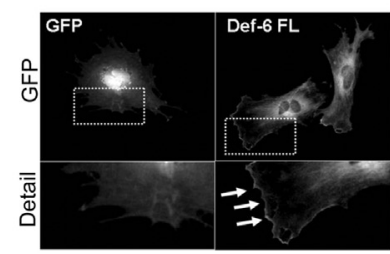

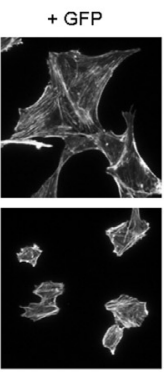

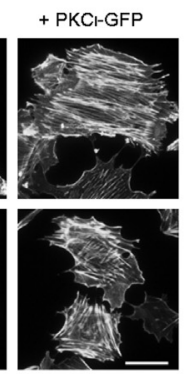

E
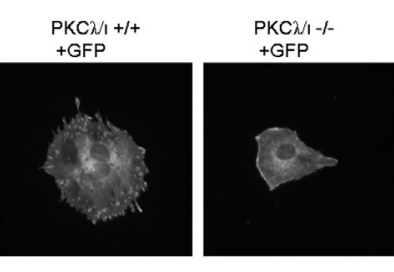

B

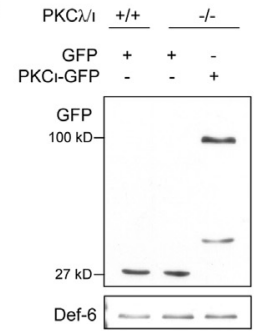

G

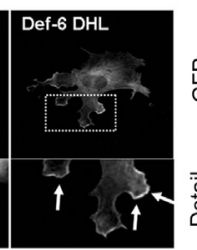

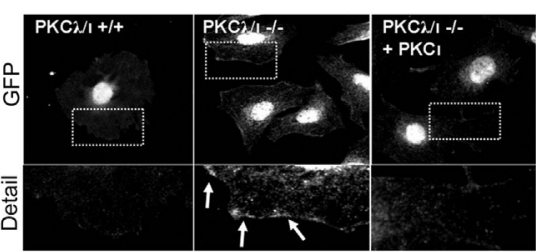

Figure 6 Overexpression of $\mathrm{PKC} \lambda / \mathrm{l}$ rescues the phenotype of $\mathrm{PKC} \lambda / \mathrm{l}^{-1-}$ podocytes. A: Real-time PCR analysis of $P K C N / \mathrm{i}^{+/+}$and $P K C \mathrm{~N} / \mathrm{i}^{-/-}$podocytes infected with adenoviral PKCI-GFP or GFP as control. Transduction with PKCI-GFP rescued the mRNA expression of $\mathrm{PKCl}$ and reduced the level of Def-6 mRNA in $P K C \mathrm{~N}_{\mathrm{i}}{ }^{-/-}$podocytes. B: Western blot analysis demonstrated overexpression of $\mathrm{PKC}_{1-}$ GFP or GFP control virus. C: Phalloidin stainings of $P K C \mathrm{~N}_{\mathrm{i}} \mathrm{i}^{+/+}$and $P K C \mathrm{M} / \mathrm{I}^{-/-}$podocytes transduced with adenoviral PKCI-GFP or GFP as control. Transduction with $\mathrm{PKCl}-\mathrm{GFP}$ rescued the actin cytoskeletal phenotype of the $P K C \mathrm{~N}_{\mathrm{i}}{ }^{-/-}$podocytes. Images were obtained using the Leica DMBL microscope $(\times 20$ magnification). Scale bar $=50$ $\mu \mathrm{m}$ (applies to all panels). D: Measurement of cell area and number of focal adhesions of vinculinstained transduced podocytes. ${ }^{* *} P<0.01,{ }^{* * *} P$ $<0.001$. E: Representative vinculin staining after virus transduction. Scale bar $=50 \mu \mathrm{m}$ (applies to all panels). F: Overexpression of Def- 6 in podocytes. In transfected podocytes, Def-6 FL GFP localizes to the cellular edges. Overexpression of constitutively active Def- 6 DHL leads to enhanced membranous localization (boxed areas in GFP views and arrows in Detail views). As control, podocytes were transfected with pEGFP-N1. G: Transduced $P K C N / \mathrm{i}^{+/+}$and $P K C N / \mathrm{i}^{-/-}$podocytes stained with an antibody against Def-6. Overexpression of PKCI reversed the enhanced membrane localization of Def- 6 in $P K C N / \mathrm{L}^{-/-}$podocytes (boxed areas in GFP views and arrows in middle Detail view). spanned by a multimolecular complex of transmembrane molecules, the slit diaphragm. Various molecules of the slit diaphragm complex, such as CD2-associated protein, bind to the actin cytoskeleton and influence the formation of actin stress fibers, ${ }^{18,36,37}$ causing the slit diaphragm to function as a signaling hub. This signaling hub consists of important tyrosine-phosphorylated signaling molecules nephrin and neph $1,{ }^{38}$ as well as several other molecules important for podocyte signal transduction. In previous studies of the PKC family of serine/threonine kinases, we found that the conventional isoform $\mathrm{PKC} \alpha$ is involved in TGF- $\beta$-induced apoptosis $^{26}$ and nephrin endocytosis ${ }^{39}$ in podocytes. We also describe that aPKC $\lambda / \mathrm{t}$ is involved in fundamental podocyte functions such as cell polarity and maintenance of the 3D podocyte architecture, and knockout of atypical isoform PKC $\zeta$ leads to no distinct renal phenotype. ${ }^{5,40,41}$ We recently have found that double knockout of both isoforms in podocytes leads to a severe developmental defect of the glomerulus, indicating a partial molecular compensation of $\mathrm{aPKC} \lambda / \mathrm{s}$ for $\mathrm{aPKC} \zeta$ in development and maintenance of the podocyte. ${ }^{7}$ The objective of the present study was to identify various molecular targets of the two aPKC isoforms, PKC $\lambda / \mathrm{t}$ and $\mathrm{PKC} \zeta$, in podocytes in vitro that explain their distinct in vivo phenotypes in the single-knockout mouse models. ${ }^{3,5,6}$

To accomplish this, we performed in vitro studies and analyzed isolated aPKC single-knockout cells in tissue culture. This revealed clear differences between the two aPKC deficiencies in podocytes. The initial finding was a striking morphologic difference between $P \mathrm{KCN}^{-/-}$podocytes and control or $P K C \zeta^{-/-}$podocytes. The observed phenotypical features included altered cell shape, increased cell movement, and a rearranged actin cytoskeleton. This altered cell shape along with the actin cytoskeletal phenotype was similar to the phenotype of podocytes described in various publications regarding the actin cytoskeleton and small GTPases. ${ }^{19,33}$ Therefore, we speculated that the 


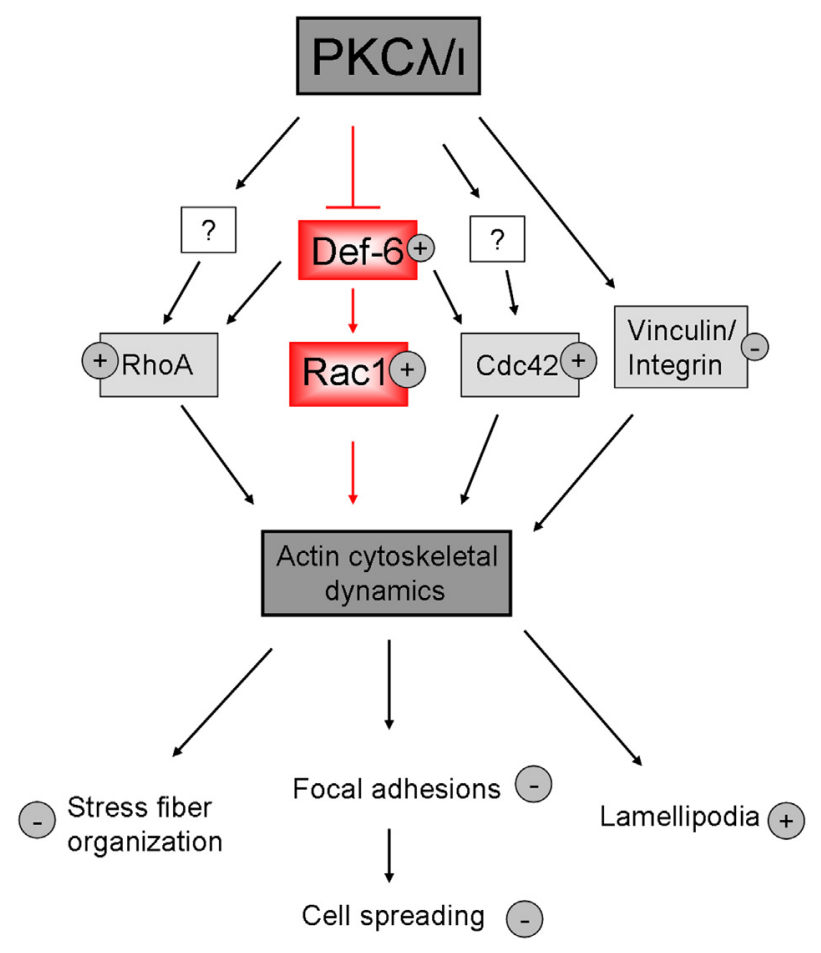

Figure 7 Proposed model for influence of $P K C \lambda / \iota$ via Def- 6 on the actin cytoskeleton. Deficiency of PKC $\lambda / \mathrm{l}$ leads to increased activation of Def- 6 and thereby to imbalance of the small GTPases, in particular Rac1. Active Rac1 influences several actin cytoskeletal-dependent mechanisms.

deficiency of aPKC $\lambda / \mathrm{t}$ podocytes might also influence the activity of small GTPases. Consistent with this hypothesis, we detected significantly more active Rac1, RhoA, and Cdc42 in the PKC $\lambda /$-deficient podocytes, with Rac1 exhibiting the most hyperactivation (Figure 2). Furthermore, we found an obvious reduction in total RhoA. It is supposed that Rho and Rac have opposing effects on the actin cytoskeleton; Rho regulates the formation of actin stress fibers, and Rac induces actin polymerization at the cellular edges to introduce lamellipodia. ${ }^{33}$ In the present study, we detected more activated RhoA and Rac1 in $P K C \mathrm{Ni}^{-1-}$ podocytes and observed fewer stress fibers and more peripheral actin. This could be explained by the strong Rac1 activation that might overrule RhoA activation. The second phenotypical feature that we analyzed in detail was the overall cell size. aPKC $\lambda /$-deficient podocytes exhibited a substantially reduced cell size and a significantly reduced number of focal adhesions. This could be explained by the changed activation pattern of the small GTPases because changed signaling of small GTPases to the actin cytoskeleton directly influences formation and turnover of focal adhesions. ${ }^{42}$ To identify distinct molecular targets, we performed a comparative microarray analysis of WT and the two aPKCdeficient podocyte cell lines. Comparison of up-regulated and down-regulated genes that were specifically changed in $\mathrm{PKC} \lambda / \mathrm{t}$-deficient but not $\mathrm{PKC} \zeta$-deficient podocytes revealed genes involved in cytoskeletal adaptor activity and filopodium formation. We identified GEF Def-6 as specifically up-regulated in $a P K C \mathrm{Ni}^{-/-}$podocytes. Def-6 is highly expressed in $\mathrm{B}$ and $\mathrm{T}$ lymphocytes, in which stimulation of the T-cell receptor leads to activation of Def- 6 and consequently to binding of $\mathrm{PIP}_{3}$ and recruitment to the immunologic synapse. ${ }^{20}$ Def-6 functions as a GEF for the small GTPases Rac1 and $\mathrm{Cdc} 42^{10}$ and has the ability to change the cellular architecture by binding to activated Rac1 in other cell types. ${ }^{43}$

Characterization of Def-6 expression in the kidney demonstrated for the first time a strong glomerular expression of Def- 6 in WT and PKC $\lambda / \mathrm{t}$-deficient mouse kidneys. In glomeruli of $\mathrm{PKC} \lambda / \mathrm{l}$-deficient mice, we detected significantly increased Def-6 expression, in particular in the podocytes. In cultured podocytes, Def- 6 was found at the cellular edges and focal adhesions, where it co-localizes with actin and vinculin (Figure 5). This is similar to earlier descriptions by Samson et $\mathrm{al}^{23}$ in NIH-3T3 fibroblasts, HeLa epithelial cells, and $\mathrm{C} 2 \mathrm{C} 12$ myoblasts. After fractionation of podocyte protein lysates, we detected an enhanced amount of endogenous Def- 6 protein in the membrane fractions, in particular in the plasma membrane of aPKC $\lambda / \mathrm{l}$-deficient podocytes. When we artificially overexpressed the full-length protein or the constitutive active form, we could confirm the observed membrane localization, in particular the active form in podocytes, much like NIH-3T3 cells in a study by Mavrakis et al. ${ }^{22}$

To demonstrate that $\mathrm{aPKC} \lambda / \mathrm{t}$ is the critical factor for the actin cytoskeletal phenotype, we re-expressed aPKC $\lambda / \mathrm{s}$ in the deficient podocytes and observed a phenotype reversal after overexpression of PKC $\lambda / \mathrm{\iota}$ (Figure 6). Further evidence for involvement of Def- 6 in $\mathrm{PKC} \lambda / \mathrm{t}$ signaling was the similar phenotype achieved in aPKC $\lambda / \mathrm{t}$-deficient and $\mathrm{WT}$ podocytes after artificial overexpression of Def-6.

Our experiments point to the balance of small GTPases and the upstream acting GEF Def- 6 as the causative factors for the detected cytoskeletal phenotype. The balance of small GTPases influenced by various GEFs and GTPaseactivating proteins is a research area of increasing importance in recent years for podocyte homeostasis. For example, Zhu et $\mathrm{al}^{19}$ have reported that activation of RhoA in podocytes induced albuminuria and focal segmental glomerulosclerosis in a transgenic mouse model. Furthermore, Akilesh et $\mathrm{al}^{16}$ have identified a Rac1 GAP, Arhgap24, that contributes to the balance of Rho and Rac signaling in podocytes and a mutant form of Arhgap24 that is associated with focal segmental glomerulosclerosis. In rat intestinal epithelial cells, expression of kinase-deficient $\mathrm{PKC} \mathrm{t}$ blocks the oncogenic Ras-mediated activation of Rac1. ${ }^{44}$ In partial contrast to these findings, we measured simultaneously increased activation of Rac1, RhoA, and $\mathrm{Cdc} 42$ in $\mathrm{PKC} \lambda / \mathrm{t}^{-1-}$ podocytes, with the most prominent effect on Rac1 activation. To our knowledge, Def-6 is the second example of molecules relevant in the immunologic synapse that demonstrate a podocyte-specific function. Similarly, CD2-associated protein was initially described as an important component of the immunologic synapse; 
knockout of CD2-associated protein did not lead to an immunologic phenotype but to a podocyte-specific phenotype with a changed actin cytoskeleton and severe proteinuria. ${ }^{45-47}$ Direct comparison with other epithelial cell types could be misleading because podocytes are a highly specialized, differentiated cell type with a unique function highly dependent on actin cytoskeleton arrangement. It is intriguing to speculate that a distinct cell-specific pathway to regulate activation of the various small GTPases in podocytes might exist. Indirect interaction of $\mathrm{Cdc} 42$ and $\operatorname{PKC} \lambda$ has already been shown by Coghlan et al, ${ }^{48}$ who found that activated $\mathrm{Cdc} 42$ and PKC $\lambda$ induce loss of stress fibers in NIH3T3 cells. In contrast, we showed that in podocytes the absence of PKC $\lambda / \mathrm{t}$ leads to fewer stress fibers and less actin accumulation at the cellular edges. In our model, we not only detected increased $\mathrm{Cdc} 42$ activity but also increased amounts of activated RhoA and in particular Rac1. This overall activation of small GTPases might influence the actin structures in several ways.

An interesting finding is that Def-6 mRNA expression was increased in microarray and real-time PCR analysis but that total Def-6 protein levels were comparable in WT and $P K C \mathrm{Ni}^{-1-}$ podocytes. However, the enhanced membrane localization of Def-6 that we detected via immunostaining and fractionation may most likely be the activated form of Def-6, which is recruited to the membrane and leads to the measured increase of activated Rac1. In this case, the protein level of Def-6 is not so important for activation of small GTPases as is recruitment of Def-6 to the plasma membrane. When Def-6 is not activated, it is kept in a closed conformation through an intramolecular interaction that inhibits its GEF function. After phosphorylation and binding to $\mathrm{PIP}_{3}$, the intramolecular interaction is released and GEF function is activated. ${ }^{20}$ There is no commercially available antibody against the activated form of Def- 6 . Thus, only membrane association can be used as an indirect indicator of Def-6 activation. Inasmuch as we found that the membrane association of Def- 6 vanishes after rescue of aPKC $\lambda / \mathrm{t}$ in deficient cells, it is highly probable that Def-6 is an important downstream effector of aPKC $\lambda / \iota$ signaling in podocytes

In conclusion, our data describe an actin cytoskeletal phenotype in $P K C \mathrm{Ni}^{-1-}$ podocytes as a consequence of disturbed balance of small GTPases, most likely caused by activation of the GEF Def-6. Differential expression of the newly identified PKC $\lambda / \iota$ target Def- 6 in glomeruli and in particular in podocytes is a finding that opens novel research avenues and describes a previously unknown pathway relevant for actin cytoskeletal dynamics in podocytes.

\section{Acknowledgments}

We thank Sandy Zachura and Germaine Puncha for technical assistance, Dr. Nathan Susnik for proofreading the manuscript, Dr. Wolfgang Ziegler for help with the
AxioObserver.Z1 microscope, and Dr. Fred Sablitzky (University of Nottingham) for providing the Def-6 constructs. Microarray experiments were performed by staff members of the service project Z02 of the Sonderforschungsbereich SFB566. All confocal images were obtained at the laser scanning facility of the Medical School of Hannover.

\section{Supplemental Data}

Supplemental material for this article can be found at http://dx.doi.org/10.1016/j.ajpath.2013.08.026.

\section{References}

1. Steinberg SF: Structural basis of protein kinase C isoform function. Physiol Rev 2008, 88:1341-1378

2. Selbie LA, Schmitz-Peiffer C, Sheng Y, Biden TJ: Molecular cloning and characterization of PKC iota, an atypical isoform of protein kinase C derived from insulin-secreting cells. J Biol Chem 1993, 268: 24296-24302

3. Soloff RS, Katayama C, Lin MY, Feramisco JR, Hedrick SM: Targeted deletion of protein kinase $\mathrm{C}$ lambda reveals a distribution of functions between the two atypical protein kinase $\mathrm{C}$ isoforms. J Immunol 2004, 173:3250-3260

4. Moeller MJ, Sanden SK, Soofi A, Wiggins RC, Holzman LB: Podocyte-specific expression of cre recombinase in transgenic mice. Genesis 2003, 35:39-42

5. Huber TB, Hartleben B, Winkelmann K, Schneider L, Becker JU, Leitges M, Walz G, Haller H, Schiffer M: Loss of podocyte aPKClambda/iota causes polarity defects and nephrotic syndrome. J Am Soc Nephrol 2009, 20:798-806

6. Leitges M, Sanz L, Martin P, Duran A, Braun U, Garcia JF, Camacho F, Diaz-Meco MT, Rennert PD, Moscat J: Targeted disruption of the zetaPKC gene results in the impairment of the NFkappaB pathway. Mol Cell 2001, 8:771-780

7. Hartleben B, Widmeier E, Suhm M, Worthmann K, Schell C, Helmstadter M, Wiech T, Walz G, Leitges M, Schiffer M, Huber TB: $\mathrm{aPKC} \lambda / \mathrm{t}$ and $\mathrm{aPKC} \zeta$ contribute to podocyte differentiation and glomerular maturation. J Am Soc Nephrol 2013, 24:253-267

8. Andrews PM, Bates SB: Filamentous actin bundles in the kidney. Anat Rec 1984, 210:1-9

9. Vasmant D, Maurice M, Feldmann G: Cytoskeleton ultrastructure of podocytes and glomerular endothelial cells in man and in the rat. Anat Rec 1984, 210:17-24

10. Ichimura K, Kurihara H, Sakai T: Actin filament organization of foot processes in rat podocytes. J Histochem Cytochem 2003, 51: $1589-1600$

11. Kriz W, Mundel P, Elger M: The contractile apparatus of podocytes is arranged to counteract GBM expansion. Contrib Nephrol 1994, 107: $1-9$

12. Pavenstädt H, Kriz W, Kretzler M: Cell biology of the glomerular podocyte. Physiol Rev 2003, 83:253-307

13. Drenckhahn D, Franke RP: Ultrastructural organization of contractile and cytoskeletal proteins in glomerular podocytes of chicken, rat, and man. Lab Invest 1988, 59:673-682

14. Kerjaschki D: Caught flat-footed: podocyte damage and the molecular bases of focal glomerulosclerosis. J Clin Invest 2001, 108:1583-1587

15. Hall A: Rho GTPases and the actin cytoskeleton. Science 1998, 279: 509-514

16. Akilesh S, Suleiman H, Yu H, Stander MC, Lavin P, Gbadegesin R, Antignac C, Pollak M, Kopp JB, Winn MP, Shaw AS: Arhgap24 inactivates Rac1 in mouse podocytes, and a mutant form is associated 
with familial focal segmental glomerulosclerosis. J Clin Invest 2011, 121:4127-4137

17. Wang L, Ellis MJ, Gomez JA, Eisner W, Fennell W, Howell DN, Ruiz P, Fields TA, Spurney RF: Mechanisms of the proteinuria induced by Rho GTPases. Kidney Int 2012, 81:1075-1085

18. van Duijn TJ, Anthony EC, Hensbergen PJ, Deelder AM, Hordijk PL: Rac1 recruits the adapter protein CMS/CD2AP to cell-cell contacts. J Biol Chem 2010, 285:20137-20146

19. Zhu L, Jiang R, Aoudjit L, Jones N, Takano T: Activation of RhoA in podocytes induces focal segmental glomerulosclerosis. J Am Soc Nephrol 2011, 22:1621-1630

20. Gupta S, Fanzo JC, Hu C, Cox D, Jang SY, Lee AE, Greenberg S, Pernis AB: T cell receptor engagement leads to the recruitment of IBP, a novel guanine nucleotide exchange factor, to the immunological synapse. J Biol Chem 2003, 278:43541-43549

21. Gupta S, Lee A, Hu C, Fanzo J, Goldberg I, Cattoretti G, Pernis AB: Molecular cloning of IBP, a SWAP-70 homologous GEF, which is highly expressed in the immune system. Hum Immunol 2003, 64: 389-401

22. Mavrakis KJ, McKinlay KJ, Jones P, Sablitzky F: DEF6, a novel PHDH-like domain protein, is an upstream activator of the Rho GTPases Rac1, Cdc42, and RhoA. Exp Cell Res 2004, 294:335-344

23. Samson T, Will C, Knoblauch A, Sharek L, von der Mark K, Burridge K, Wixler V: Def-6, a guanine nucleotide exchange factor for Rac1, interacts with the skeletal muscle integrin chain alpha7A and influences myoblast differentiation. J Biol Chem 2007, 282: $15730-15742$

24. Helfrich I, Schmitz A, Zigrino P, Michels C, Haase I, le Bivic A, Leitges M, Niessen CM: Role of aPKC isoforms and their binding partners Par3 and Par6 in epidermal barrier formation. J Invest Dermatol 2007, 127:782-791

25. Jat PS, Noble MD, Ataliotis P, Tanaka Y, Yannoutsos N, Larsen L, Kioussis D: Direct derivation of conditionally immortal cell lines from an H-2Kb-tsA58 transgenic mouse. Proc Natl Acad Sci U S A 1991, 88:5096-5100

26. Tossidou I, Starker G, Kruger J, Meier M, Leitges M, Haller H, Schiffer M: PKC-alpha modulates TGF-beta signaling and impairs podocyte survival. Cell Physiol Biochem 2009, 24:627-634

27. Mundel P, Reiser J, Zúñiga Mejía BA, Pavenstädt H, Davidson GR, Kriz W, Zeller R: Rearrangements of the cytoskeleton and cell contacts induce process formation during differentiation of conditionally immortalized mouse podocyte cell lines. Exp Cell Res 1997, 236: $248-258$

28. Marg S, Winkler U, Sestu M, Himmel M, Schönherr M, Bär J, Mann A, Moser M, Mierke CT, Rottner K, Blessing M, Hirrlinger J, Ziegler WH: The vinculin-DeltaIn20/21 mouse: characteristics of a constitutive, actin-binding deficient splice variant of vinculin. PLoS One 2010, 5:e11530

29. Mundel P, Gilbert P, Kriz W: Podocytes in glomerulus of rat kidney express a characteristic 44 KD protein. J Histochem Cytochem 1991, 39:1047-1056

30. Mundlos S, Pelletier J, Darveau A, Bachmann M, Winterpacht A, Zabel B: Nuclear localization of the protein encoded by the Wilms' tumor gene WT1 in embryonic and adult tissues. Development 1993, 119:1329-1341
31. Sander EE, ten Klooster JP, van Delft S, van der Kammen RA, Collard JG: Rac downregulates Rho activity: reciprocal balance between both GTPases determines cellular morphology and migratory behavior. J Cell Biol 1999, 147:1009-1022

32. Bernards A: GAPs galore! a survey of putative Ras superfamily GTPase activating proteins in man and Drosophila. Biochim Biophys Acta 2003, 1603:47-82

33. Hall A: Rho GTPases and the control of cell behaviour. Biochem Soc Trans 2005, 33(Pt 5):891-895

34. Ziegler WH, Liddington RC, Critchley DR: The structure and regulation of vinculin. Trends Cell Biol 2006, 16:453-460

35. Etienne-Manneville S, Hall A: Integrin-mediated activation of Cdc42 controls cell polarity in migrating astrocytes through PKCzeta. Cell 2001, 106:489-498

36. Asanuma K, Yanagida-Asanuma E, Faul C, Tomino Y, Kim K, Mundel P: Synaptopodin orchestrates actin organization and cell motility via regulation of RhoA signalling. Nat Cell Biol 2006, 8: 485-491

37. Yuan H, Takeuchi E, Salant DJ: Podocyte slit-diaphragm protein nephrin is linked to the actin cytoskeleton. Am J Physiol Renal Physiol 2002, 282:F585-F591

38. Benzing T: Signaling at the slit diaphragm. J Am Soc Nephrol 2004 , 15:1382-1391

39. Tossidou I, Teng B, Menne J, Shushakova N, Park JK, Becker JU, Modde F, Leitges M, Haller H, Schiffer M: Podocytic PKC-alpha is regulated in murine and human diabetes and mediates nephrin endocytosis. PLoS One 2010, 5:e10185

40. Hartleben B, Schweizer H, Lübben P, Bartram MP, Möller CC, Herr R, Wei C, Neumann-Haefelin E, Schermer B, Zentgraf H, Kerjaschki D, Reiser J, Walz G, Benzing T, Huber TB: Neph-Nephrin proteins bind the Par3-Par6-atypical protein kinase C (aPKC) complex to regulate podocyte cell polarity. J Biol Chem 2008, 283:23033-23038

41. Simons M, Hartleben B, Huber TB: Podocyte polarity signalling. Curr Opin Nephrol Hypertens 2009, 18:324-330

42. Rottner K, Stradal TE: Actin dynamics and turnover in cell motility. Curr Opin Cell Biol 2011, 23:569-578

43. Oka T, Ihara S, Fukui Y: Cooperation of DEF6 with activated Rac in regulating cell morphology. J Biol Chem 2007, 282:2011-2018

44. Murray NR, Jamieson L, Yu W, Zhang J, Gokmen-Polar Y, Sier D, Anastasiadis P, Gatalica Z, Thompson EA, Fields AP: Protein kinase Ciota is required for Ras transformation and colon carcinogenesis in vivo. J Cell Biol 2004, 164:797-802

45. Lehtonen S, Zhao F, Lehtonen E: CD2-associated protein directly interacts with the actin cytoskeleton. Am J Physiol Renal Physiol 2002, 283:F734-F743

46. Shih NY, Li J, Karpitskii V, Nguyen A, Dustin ML, Kanagawa O, Miner JH, Shaw AS: Congenital nephrotic syndrome in mice lacking CD2-associated protein. Science 1999, 286:312-315

47. Dustin ML, Olszowy MW, Holdorf AD, Li J, Bromley S, Desai N, Widder P, Rosenberger F, van der Merwe PA, Allen PM, Shaw AS: A novel adaptor protein orchestrates receptor patterning and cytoskeletal polarity in T-cell contacts. Cell 1998, 94:667-677

48. Coghlan MP, Chou MM, Carpenter CL: Atypical protein kinases Clambda and -zeta associate with the GTP-binding protein Cdc42 and mediate stress fiber loss. Mol Cell Biol 2000, 20:2880-2889 